\title{
On Analytic Vectors for Unitary Representations of Infinite Dimensional Lie Groups
}

\author{
Karl-Hermann Neeb* ${ }^{\dagger}$
}

October 6, 2010

\begin{abstract}
Let $G$ be a connected and simply connected Banach-Lie group or, more generally, a BCH-Lie group. On the complex enveloping algebra $U_{\mathbb{C}}(\mathfrak{g})$ of its Lie algebra $\mathfrak{g}$ we define the concept of an analytic functional and show that every positive analytic functional $\lambda$ is integrable in the sense that it is of the form $\lambda(D)=\langle\mathrm{d} \pi(D) v, v\rangle$ for an analytic vector $v$ of a unitary representation of $G$. On the way to this result we derive criteria for the integrability of $*$-representations of infinite dimensional Lie algebras of unbounded operators to unitary group representations.

For the matrix coefficient $\pi^{v, v}(g)=\langle\pi(g) v, v\rangle$ of a vector $v$ in a unitary representation of an analytic Fréchet-Lie group $G$ we show that $v$ is an analytic vector if and only if $\pi^{v, v}$ is analytic in an identity neighborhood. Combining this insight with the results on positive analytic functionals, we derive that every local positive definite analytic function on a 1-connected Fréchet-BCH-Lie group $G$ extends to a global analytic function.

Keywords: infinite dimensional Lie group, unitary representation, positive definite function, analytic vector, integrability of Lie algebra representations. MSC2000: 22E65, 22E45.
\end{abstract}

\section{Contents}

1 Introduction $\quad 2$

2 Locally convex Lie groups 6

3 Analytic functionals

4 Analytic vectors and continuity of Lie algebra representations 14

5 Analytic vectors of unitary representations 17

${ }^{*}$ Department Mathematik, FAU Erlangen-Nürnberg, Bismarckstrasse 1 1/2, 91054-Erlangen, Germany; neeb@mi.uni-erlangen.de

†Supported by DFG-grant NE 413/7-1, Schwerpunktprogramm "Darstellungstheorie". 


\section{Introduction}

Let $G$ be an analytic Lie group modeled on a locally convex space and $(\pi, \mathcal{H})$ be a unitary representation of $G$ 还 We call a vector $v \in \mathcal{H}$ smooth, resp., analytic if its orbit map $\pi^{v}: G \rightarrow \mathcal{H}, g \mapsto \pi(g) v$ is smooth, resp., analytic. We write $\mathcal{H}^{\infty}$, resp., $\mathcal{H}^{\omega}$ for the subspace of smooth, resp., analytic vectors. Analytic vectors for unitary representations of finite dimensional Lie groups play an important role in the translation process between the representation $\pi$ of a connected Lie group $G$ and the derived representation $\mathrm{d} \pi: \mathfrak{g} \rightarrow \operatorname{End}\left(\mathcal{H}^{\infty}\right)$ of its Lie algebra $\mathfrak{g}$. In particular, for a subspace $E \subseteq \mathcal{H}$ consisting of smooth vectors and invariant under $\mathfrak{g}$, we can only conclude its invariance under $G$ if the set of analytic vectors in $E$ is dense (Wa72, Cor. 4.4.5.5]). The key point in the argument is the Taylor expansion of an analytic function $f: G \rightarrow E$ with values in a Banach space $E$ near the identity:

$$
f(\exp X)=\sum_{n=0}^{\infty} \frac{1}{n !}\left(X^{n} f\right)(\mathbf{1}),
$$

where the elements $X \in \mathfrak{g}$ acts as a left invariant differential operator on $C^{\infty}(G, E)$. The summands in this expansion define a linear map $T(f): U(\mathfrak{g}) \rightarrow E, D \mapsto(D f)(\mathbf{1})$. Our motivation for this paper was to understand which functionals on $U(\mathfrak{g})$ correspond in this sense to a matrix coefficient $\pi^{v, v}(g)=\langle\pi(g) v, v\rangle$ of an analytic vector $v$ for a unitary representation $(\pi, \mathcal{H})$ of $G$.

As we shall see below, all this concerns only representations $(\pi, \mathcal{H})$ for which $\mathcal{H}^{\omega}$ is dense. For finite dimensional Lie groups, this is always the case for continuous unitary representations on Hilbert spaces. Building on previous work of Harish-Chandra on semisimple groups, Cartier and Dixmier prove in CD58 the density of $\mathcal{H}^{\omega}$ for unitary representations and continuous Banach representations which are bounded on a certain discrete central subgroup. The general case was obtained by Nelson ([Nel59, Thm. 4]) and Gårding (Ga60]) by convolution with heat kernels (cf. Wa72, Sect. 4.4.5]). For generalizations of these density results to representations on locally convex spaces, we refer to Mo72.

For infinite dimensional Lie groups a continuous unitary representation need not possess any differentiable vector. Refining a construction from [BN08], we have shown in Ne10 that the continuous unitary representation of the abelian Banach-Lie group $G=\left(L^{p}([0,1], \mathbb{R}),+\right), p \geq 1$, on the Hilbert space $\mathcal{H}=L^{2}([0,1], \mathbb{C})$ by $\pi(g) f=e^{i g} f$ satisfies $\mathcal{H}^{\infty}=\{0\}$. For $p=1$, we thus obtain a continuous representation with no non-zero $C^{1}$-vector, and for $p=2 k$, the space of $C^{k}$-vectors is non-zero, but there is no non-zero $C^{k+1}$-vector.

As a consequence of these examples, one cannot expect analytic vectors to form a dense subspace for infinite dimensional Lie groups, and not even for Banach-Lie

\footnotetext{
${ }^{1} \mathrm{Cf}$. $[\mathrm{Ne} 06$ for a survey on locally convex Lie theory and Section 2 for basic definitions.
} 
groups. Up to now, only a few classes of groups are known for which $\mathcal{H}^{\omega}$ is dense for every continuous unitary representation. If $G$ is the additive group of a nuclear locally convex space, this follows from the Bochner-Minlos Theorem and the regularity of the corresponding measures (cf. Heg72, Thm. 2.11] and [Sa91, Sect. 2.5] for the special case of the countably dimensional vector group $\left.G=\mathbb{R}^{(\mathbb{N})}\right)$. One also has a corresponding result for Heisenberg groups: Let $V$ be a locally convex space, endowed with a continuous positive definite scalar product. Then we define the corresponding Heisenberg group by

$\operatorname{Heis}(V)=\mathbb{R} \times V \times V, \quad(z, v, w)\left(z^{\prime}, v^{\prime}, w^{\prime}\right):=\left(z+z^{\prime}+\frac{1}{2}\left(\left\langle v, w^{\prime}\right\rangle-\left\langle v^{\prime}, w\right\rangle\right), v+v^{\prime}, w+w^{\prime}\right)$.

In Heg72 Hegerfeldt shows that, if $V$ is separable, barrelled and nuclear, for any continuous unitary representation $(\pi, \mathcal{H})$ of $\operatorname{Heis}(V)$, the space $\mathcal{H}^{\omega}$ is dense. He even proves the existence of a dense subspace $\mathcal{D} \subseteq \mathcal{H}^{\omega}$ with the property that for every $v \in \mathcal{D}$ the orbit map $\pi^{v}(g)=\pi(g) v$ extends to a holomorphic map Heis $(V)_{\mathbb{C}} \rightarrow \mathcal{H}$ (see also $\operatorname{Re} 69$ for the special case $V=\mathbb{R}^{(\mathbb{N})}$ and certain Banach completions). Further results for direct limits of finite dimensional matrix groups can be found in Sa91, Sect. 6.4].

In this paper we are concerned with two aspects of analytic vectors for representations of a 1-connected Lie group $G$ with Lie algebra $\mathfrak{g}$ :

(IR) The integrability problem for Lie algebra representations: Given a $*$-representation $(\rho, \mathcal{D})$ of $\mathfrak{g}$ on a pre-Hilbert space $\mathcal{D}$, when is there a continuous unitary representation $(\pi, \mathcal{H})$ of $G$ on the completion $\mathcal{H}$ of $\mathcal{D}$ with $\mathcal{D} \subseteq \mathcal{H}^{\infty}$ and $\rho(x)=\left.\mathrm{d} \pi(x)\right|_{\mathcal{D}}$ for $x \in \mathfrak{g}$ ? The criteria we discuss here are based on a rich supply of analytic vectors.

(IF) The integrability problem for functionals on $U(\mathfrak{g})$ : Which linear functionals $\lambda: U_{\mathbb{C}}(\mathfrak{g}):=U(\mathfrak{g})_{\mathbb{C}} \rightarrow \mathbb{C}$ are integrable in the sense that there exists a continuous unitary representation $(\pi, \mathcal{H})$ of $G$ and a smooth vector $v \in \mathcal{H}^{\infty}$ with $\lambda(D)=$ $\langle\mathrm{d} \pi(D) v, v\rangle$ for $D \in U(\mathfrak{g})$ ?

As far as we know, the present knowledge on (IR) is limited to finite dimensional Lie algebras and groups. It is based on Nelson's Theorem, asserting that a symmetric operator $A$ on a Hilbert space $\mathcal{H}$ is essentially selfadjoint if it has a dense subspace of vectors $v$ which are analytic in the sense that $\sum_{n} \frac{\left\|A^{n} v\right\| t^{n}}{n !}<\infty$ holds for some $t>0$. In view of Stone's Theorem, for such operators $A$, the selfadjoint closure $\bar{A}$ generates a unitary one-parameter group $e^{i t \bar{A}}$. The first generalization of this result to finite dimensional Lie algebras $\mathfrak{g}$ can be found in FSSS72, where it is shown that any representation $\rho: \mathfrak{g} \rightarrow \operatorname{End}(\mathcal{D})$ by skew-symmetric operators on a dense subspace $\mathcal{D}$ of a Hilbert space $\mathcal{H}$ can be integrated to a unitary group representation if $\mathcal{D}$ consists of analytic vectors for the operators $\rho\left(x_{1}\right), \ldots, \rho\left(x_{n}\right)$, where $x_{1}, \ldots, x_{n}$ is a basis of g. In [Si72] Simon generalizes this result to the situation where $x_{1}, \ldots, x_{n}$ are only required to generate $\mathfrak{g}$ as a Lie algebra. These integrability criteria for representations of finite dimensional Lie algebras by unbounded operators generalize to representations on Banach spaces (cf. GJ83, see also Jo88, Cor. A.4]). We recommend Section 5 in Mag92 for a detailed discussion of analytic vectors and integrability conditions 
for finite dimensional Lie algebras. One of our main results is a solution of (IR) for Banach-Lie algebras asserting the integrability of $\rho$ if $\mathcal{D}$ consists of analytic vectors.

Problem (IF) is an infinite dimensional variant of the classical (non-commutative) moment problem. Since smoothness of a vector $v \in \mathcal{H}$ is equivalent to smoothness of the corresponding positive definite function $\pi^{v, v}(g)=\langle\pi(g) v, v\rangle$ ([Ne10, Thm. 7.2]), the GNS construction implies that (IF) is equivalent to the existence of a smooth positive definite function $\varphi$ with $\lambda(D)=(D \varphi)(\mathbf{1})$ for $D \in U_{\mathbb{C}}(\mathfrak{g})$.

If $\mathfrak{g}$ is finite dimensional and $x_{1}, \ldots, x_{k}$ is a basis of $\mathfrak{g}$, then every linear functional $\lambda \in U_{\mathbb{C}}(\mathfrak{g})^{*}$ defines a function

$$
s_{\lambda}: \mathbb{N}_{0}^{k} \rightarrow \mathbb{C}, \quad \alpha \mapsto \lambda\left(x^{\alpha}\right), \quad x^{\alpha}:=x_{1}^{\alpha_{1}} \cdots x_{k}^{\alpha_{k}} .
$$

In view of the Poincaré-Birkhoff-Witt Theorem, the correspondence between linear functionals on $U_{\mathbb{C}}(\mathfrak{g})$ and functions on $\mathbb{N}_{0}^{k}$ is one-to-one. The non-commutative moment problem is to decide for $s: \mathbb{N}_{0}^{k} \rightarrow \mathbb{C}$ when the corresponding functional $\lambda_{s} \in U_{\mathbb{C}}(\mathfrak{g})^{*}$ is integrable in the sense of (IF) (cf. [Sa91, Sect. 12.2]). For $G=\mathbb{R}^{k}$ this specializes to the classical moment problem which, for $\left(s_{\alpha}\right)_{\alpha \in \mathbb{N}_{0}^{k}}$, asks for a bounded positive measure $\mu$ on $\mathbb{R}^{k}$ with

$$
s_{\alpha}=\int_{\mathbb{R}^{k}} x^{\alpha} \mathrm{d} \mu(x) \quad \text { for } \quad \alpha \in \mathbb{N}_{0}^{k} .
$$

Here we use Bochner's Theorem which says that the Fourier transform $\mu \mapsto \widehat{\mu}$ defines a bijection between bounded positive Borel measures on $\mathbb{R}^{k}$ and continuous positive definite functions on $\mathbb{R}^{k}$ (see Ak65] for more on the classical moment problem). For results concerning moment problems for nuclear spaces, we refer to [Sch90, Thm. 12.5.2] and the original papers BY75, Heg75.

An obvious necessary condition for the solvability of (IF) is that $\lambda$ must be a positive functional on the $*$-algebra $U_{\mathbb{C}}(\mathfrak{g})$ in the sense that $\lambda\left(D^{*} D\right) \geq 0$ for $D \in$ $U_{\mathbb{C}}(\mathfrak{g})$, but in general this condition is not sufficient for integrability. For $G=\mathbb{R}^{k}$, a necessary and sufficient condition for integrability is non-negativity of $\lambda$ on positive polynomials in $U(\mathfrak{g}) \cong \mathbb{R}\left[x_{1}, \ldots, x_{k}\right]$, which is stronger than positivity because, for $k \geq 2$, not every non-negative polynomial is a sum of squares (Sch90, Ex. 2.6.11], BCR84, Sects. 6.1/2]). In the light of these results, it is quite remarkable that, for $G=\mathbb{R}^{k}$, positivity of $\lambda$ plus estimates on the $s_{\alpha}$ which ensure convergence of the series $\sum_{\alpha} \frac{1}{\alpha !} x^{\alpha} s_{\alpha}$ in a zero-neighborhood of $\mathbb{R}^{k}$ imply unique solvability of (IF) (Sa91, Lemma 12.2.1], [Sh96, Thm. II.12.7]). A non-commutative variant of this result for Heisenberg groups can be found in [Sa91, Thm. 12.2.3].

For the solvability of (IF) for a connected Lie group $G$, Schmüdgen shows in Sch78, Thm. 5.1] that $\lambda \in U_{\mathbb{C}}(\mathrm{E}(G))^{*}$ is integrable if and only if it is strongly positive in the sense that $\lambda(D) \geq 0$ whenever $\mathrm{d} \pi(D) \geq 0$ holds for all unitary representations $(\pi, \mathcal{H})$ of $G$. He also shows in $[\mathrm{Sch} 78$, Thm. 4.1] that for $G \neq \mathbb{R}$ there exist positive functionals which are not strongly positive. With respect to (IR) for finite dimensional groups, one has a necessary and sufficient criterion in terms of so-called complete positivity (Sch78, Sch90, Thm. 11.4.2], Po74, Thm. 4.5]). Here we are only concerned with the unique solvability of (IF) for positive functionals which are "analytic" in the sense that they satisfy certain estimates. 
The plan of this paper is as follows. After recalling the basic concepts concerning infinite dimensional Lie groups and analytic functions in Section 2, we introduce in Section 3 the concept of an analytic linear map $\beta: U_{\mathbb{C}}(\mathfrak{g}) \rightarrow E$ to a Banach space $E$. These are linear maps for which the series $\sum_{n=0}^{\infty} \frac{\beta\left(x^{n}\right)}{n !}$ converges on a 0-neighborhood of $\mathfrak{g}$. The main result of Section 3 is Theorem 3.6 which asserts that, for any continuous seminorm $p$ on $\mathfrak{g}$ which is submultiplicative in the sense that $p([x, y]) \leq p(x) p(y)$ for $x, y \in \mathfrak{g}$, the space of those analytic linear maps for which $\sum_{n} \frac{\beta\left(x^{n}\right)}{n !}$ converges uniformly on the $r$-ball with respect to $p$ is invariant under the left and right regular representation of $U(\mathfrak{g})$ on $\operatorname{Hom}(U(\mathfrak{g}), E)$.

In Section 4 we turn to representations $\rho: \mathfrak{g} \rightarrow \operatorname{End}(V)$ of a Lie algebras $\mathfrak{g}$ on a normed space $V$. We call such a representation strongly continuous if the orbit maps $\rho^{v}: \mathfrak{g} \rightarrow V, x \mapsto \rho(x) v$ are continuous and say that $v \in V$ is analytic if $\sum_{n} \frac{\left\|\rho(x)^{n} v\right\|}{n !}$ converges on some 0 -neighborhood of $\mathfrak{g}$. If $\mathfrak{g}$ is a Baire space, $v$ is analytic if and only if it is an analytic vector for all operators $\rho(x)$ separately (Proposition 4.10). In Section 5 we eventually turn to unitary representations of an analytic Lie group $G$ with Lie algebra $\mathfrak{g}$ by showing that $v \in \mathcal{H}$ is analytic if and only if the matrix coefficient $\pi^{v, v}(g)=\langle\pi(g) v, v\rangle$ is analytic in some 1-neighborhood. This reduces the analyticity condition for $v$ to a local requirement on a scalar-valued function. In Section 6 we proceed to the infinitesimal picture with the observation that for a $*$-representation of $\mathfrak{g}$ on a pre-Hilbert space $\mathcal{D}$, an element $v \in \mathcal{D}$ is an analytic vector if and only if the linear functional $\rho^{v, v}(D):=\langle\rho(D) v, v\rangle$ on $U(\mathfrak{g})$ is analytic. The main results of Section 6 are integrability results for $*$-representations on pre-Hilbert spaces. Theorem 6.8 asserts that for a 1-connected Banach-Lie group $G$ a $*$-representation $(\rho, \mathcal{D})$ of $\mathfrak{g}$ is integrable if $\mathcal{D}$ consists of analytic vectors. Refining this result for the larger class of BCH-Lie groups, we derive in particular, that every positive analytic functional $\lambda$ on the $*-$ algebra $U_{\mathbb{C}}(\mathfrak{g})$ is integrable in the sense of (IF). We conclude Section 6 with a variant of the integrability theorem on Lie algebra representations that does not require an a priori given Lie group $G$. The interest in this refinement lies in the fact that not every Banach-Lie algebra is the Lie algebra of some Banach-Lie group ([EK64]). The class of BCH-Lie groups is substantially larger than the class of Banach-Lie groups. It contains in particular all (projective limits of) nilpotent Lie groups and groups of smooth maps on compact manifolds with values in Banach-Lie groups (see GN10 for more details). In particular, all Heisenberg groups are $\mathrm{BCH}$.

From the integrability of positive analytic functionals on $U_{\mathbb{C}}(\mathfrak{g})$, we further derive in Section 7 that any germ of an analytic positive definite function $\varphi$ on a 1-connected $\mathrm{BCH}-\mathrm{Lie}$ group extends uniquely to a global analytic positive definite function (Theorem 7.3).

An interesting issue that deserves to be pursued in the future is that the concept of an analytic vector makes sense for any representation $(\rho, V)$ of a locally convex Lie algebra $\mathfrak{g}$ on a normed space $V$, even when there is no analytic Lie group $G$ with Lie algebra $\mathfrak{g}$. This may open a door to applications for large classes of non-analytic Lie groups such as diffeomorphism groups of compact smooth manifolds.

In his recent paper Me10, S. Merigon generalizes our Integrability Theorem 5.2 for simply connected Banach-Lie groups in the sense that he only requires the existence of a direct sum decomposition $\mathfrak{g}=\mathfrak{g}_{1} \oplus \cdots \oplus \mathfrak{g}_{n}$ into closed subspaces, such that all 
vectors are analytic for every $x \in \mathfrak{g}_{j}, j=1, \ldots, n$. We expect the results in this paper and $\mathrm{Me10}$, to be the key tools to generalize the theory of analytic extension of unitary group representations ([LM75], [Jo86, Jo87, O190, O191], [Ne00]) to the context of Banach-Lie groups.

\section{Locally convex Lie groups}

In this section we briefly recall the basic concepts related to infinite dimensional Lie groups and calculus on locally convex spaces.

Definition 2.1 (a) Let $E$ and $F$ be locally convex spaces, $U \subseteq E$ open and $f: U \rightarrow F$ a map. Then the derivative of $f$ at $x$ in the direction $h$ is defined as

$$
\mathrm{d} f(x)(h):=\left(\partial_{h} f\right)(x):=\left.\frac{d}{d t}\right|_{t=0} f(x+t h)=\lim _{t \rightarrow 0} \frac{1}{t}(f(x+t h)-f(x))
$$

whenever it exists. The function $f$ is called differentiable at $x$ if $\mathrm{d} f(x)(h)$ exists for all $h \in E$. It is called continuously differentiable, if it is differentiable at all points of $U$ and

$$
\mathrm{d} f: U \times E \rightarrow F, \quad(x, h) \mapsto \mathrm{d} f(x)(h)
$$

is a continuous map. Note that this implies that the maps $\mathrm{d} f(x)$ are linear (cf. GN10, Lemma 2.2.14]). The map $f$ is called a $C^{k}-\operatorname{map}, k \in \mathbb{N} \cup\{\infty\}$, if it is continuous, the iterated directional derivatives

$$
\mathrm{d}^{j} f(x)\left(h_{1}, \ldots, h_{j}\right):=\left(\partial_{h_{j}} \cdots \partial_{h_{1}} f\right)(x)
$$

exist for all integers $j \leq k, x \in U$ and $h_{1}, \ldots, h_{j} \in E$, and all maps $\mathrm{d}^{j} f: U \times E^{j} \rightarrow F$ are continuous. As usual, $C^{\infty}$-maps are called smooth.

(b) If $E$ and $F$ are complex locally convex spaces, then $f$ is called complex analytic if it is continuous and for each $x \in U$ there exists a 0-neighborhood $V$ with $x+V \subseteq U$ and continuous homogeneous polynomials $\beta_{k}: E \rightarrow F$ of degree $k$ such that for each $h \in V$ we have

$$
f(x+h)=\sum_{k=0}^{\infty} \beta_{k}(h),
$$

as a pointwise limit ( $(\mathrm{BS71b})$. The map $f$ is called holomorphic if it is $C^{1}$ and for each $x \in U$ the map d $f(x): E \rightarrow F$ is complex linear (cf. [Mil84, p. 1027]). If $F$ is sequentially complete, then $f$ is holomorphic if and only if it is complex analytic ( BS71b, Ths. 3.1, 6.4]).

(c) If $E$ and $F$ are real locally convex spaces, then we call a map $f: U \rightarrow F$, $U \subseteq E$ open, real analytic or a $C^{\omega}$-map, if for each point $x \in U$ there exists an open neighborhood $V \subseteq E_{\mathbb{C}}$ and a holomorphic map $f_{\mathbb{C}}: V \rightarrow F_{\mathbb{C}}$ with $\left.f_{\mathbb{C}}\right|_{U \cap V}=\left.f\right|_{U \cap V}$ (cf. Mil84). The advantage of this definition, which differs from the one in BS71b, is that it also works nicely for non-complete spaces. Any analytic map is smooth, and the corresponding chain rule holds without any condition on the underlying spaces, which is the key to the definition of analytic manifolds (see G102 for details). 
Once the concept of a smooth function between open subsets of locally convex spaces is established (cf. [Ne06, Mil84, GN10]), it is clear how to define a locally convex smooth manifold. A (locally convex) Lie group $G$ is a group equipped with a smooth manifold structure modeled on a locally convex space for which the group multiplication and the inversion are smooth maps. We write $\mathbf{1} \in G$ for the identity element and $\lambda_{g}(x)=g x$, resp., $\rho_{g}(x)=x g$ for the left, resp., right multiplication on $G$. Then each $x \in T_{1}(G)$ corresponds to a unique left invariant vector field $x_{l}$ with $x_{l}(g):=T_{\mathbf{1}}\left(\lambda_{g}\right) x, g \in G$. The space of left invariant vector fields is closed under the Lie bracket of vector fields, hence inherits a Lie algebra structure. In this sense we obtain on $\mathfrak{g}:=T_{\mathbf{1}}(G)$ a continuous Lie bracket which is uniquely determined by $[x, y]_{l}=\left[x_{l}, y_{l}\right]$ for $x, y \in \mathfrak{g}$. We shall also use the functorial notation $\mathrm{E}(G):=(\mathfrak{g},[\cdot, \cdot])$ for the Lie algebra of $G$ and, accordingly, $\mathrm{E}(\varphi)=T_{1}(\varphi): \mathrm{L}\left(G_{1}\right) \rightarrow \mathrm{L}\left(G_{2}\right)$ for the Lie algebra morphism associated to a morphism $\varphi: G_{1} \rightarrow G_{2}$ of Lie groups. Then $\mathrm{E}$ defines a functor from the category of locally convex Lie groups to the category of locally convex Lie algebras, i.e., locally convex spaces, endowed with continuous Lie brackets. The adjoint action of $G$ on $\mathrm{E}(G)$ is defined by $\operatorname{Ad}(g):=\mathrm{E}\left(c_{g}\right)$, where $c_{g}(x)=g x g^{-1}$ is the conjugation map. The adjoint action is smooth and each $\operatorname{Ad}(g)$ is a topological isomorphism of $\mathfrak{g}$. If $\mathfrak{g}$ is a Fréchet, resp., a Banach space, then $G$ is called a Fréchet-, resp., a Banach-Lie group.

A smooth map $\exp _{G}: \mathfrak{g} \rightarrow G$ is called an exponential function if each curve $\gamma_{x}(t):=$ $\exp _{G}(t x)$ is a one-parameter group with $\gamma_{x}^{\prime}(0)=x$. Not every infinite dimensional Lie group has an exponential function ([Ne6, Ex. II.5.5]), but exponential functions are unique whenever they exist. A Lie group $G$ is said to be locally exponential if it has an exponential function for which there is an open 0-neighborhood $U$ in $\mathrm{E}(G)$ mapped diffeomorphically by $\exp _{G}$ onto an open subset of $G$. If, in addition, $G$ is analytic and the exponential function is an analytic local diffeomorphism in 0 , then $G$ is called a $B C H$-Lie group. Then the Lie algebra $\mathrm{L}(G)$ is a $B C H$-Lie algebra (for Baker-Campbell-Hausdorff), i.e., there exists an open 0-neighborhood $U \subseteq \mathfrak{g}$ such that for $x, y \in U$ the $\mathrm{BCH}$ series

$$
x * y=x+y+\frac{1}{2}[x, y]+\cdots
$$

converges and defines an analytic function $U \times U \rightarrow \mathfrak{g},(x, y) \mapsto x * y$. The class of $\mathrm{BCH}-\mathrm{Lie}$ groups contains in particular all Banach-Lie groups ([Ne06, Prop. IV.1.2]).

If $\pi: G \rightarrow \mathrm{GL}(V)$ is a representation of $G$ on a locally convex space $V$, the exponential function permits us to associate to each element $x$ of the Lie algebra a one-parameter group $\pi_{x}(t):=\pi\left(\exp _{G} t x\right)$. We therefore assume in the following that $G$ has an exponential function.

\section{Analytic functionals}

In the following $\mathfrak{g}$ denotes a real locally convex Lie algebra and $U(\mathfrak{g})$ its enveloping algebra. The continuity of the Lie bracket on $\mathfrak{g}$ means that, for every continuous seminorm $p$ on $\mathfrak{g}$, there exists a continuous seminorm $\widetilde{p}$ on $\mathfrak{g}$ satisfying

$$
p([x, y]) \leq \widetilde{p}(x) \widetilde{p}(y) \quad \text { for } \quad x, y \in \mathfrak{g} .
$$


If this relation holds with $\widetilde{p}=p$, then we call $p$ is submultiplicative. If $\mathfrak{g}$ is a Banach-Lie algebra, then we assume that the norm on $\mathfrak{g}$ is submultiplicative

$$
\|[x, y]\| \leq\|x\| \cdot\|y\| \quad \text { for } \quad x, y \in \mathfrak{g},
$$

which can always be achieved by replacing it by a suitable multiple.

In this section we introduce for each continuous submultiplicative seminorm $p$ on $\mathfrak{g}$ the concept of a $p$-analytic linear map $\beta$ on the enveloping algebra $U(\mathfrak{g})$ with values in a Banach space $E$ and define its radius of convergence $R_{\beta, p}$. The main result of this section is Theorem [3.6. asserting that the subspace of elements $\beta$ with $R_{\beta, p} \geq r$ is invariant under the left and right regular action of $U(\mathfrak{g})$. This applies in particular to the norm $p(x)=\|x\|$ of a Banach-Lie algebra.

Definition 3.1 Let $V$ be a locally convex space and $W$ be a normed space. We write $\operatorname{Mult}^{n}(V, W)$ for the space of continuous $W$-valued $n$-linear maps on $V^{n}$ and $\operatorname{Sym}^{n}(V, W)$ for the subspace of symmetric $n$-linear maps. We also write $\mathcal{P}(V)$ for the set of continuous seminorms on $V$.

(a) For an $n$-linear map $\beta: V^{n} \rightarrow W$ and $p \in \mathcal{P}(V)$, we define

$$
\|\beta\|_{p}:=\sup \left\{\left\|\beta\left(v_{1}, \ldots, v_{n}\right)\right\|: v_{1}, \ldots, v_{n} \in V, p\left(v_{i}\right) \leq 1\right\} \in[0, \infty] .
$$

Then $\beta$ is continuous with respect to the topology on $V$ defined by $p$ if and only if $\|\beta\|_{p}<\infty$, and we have

$$
\left\|\beta\left(v_{1}, \ldots, v_{n}\right)\right\| \leq\|\beta\|_{p} p\left(v_{1}\right) \cdots p\left(v_{n}\right) \quad \text { for } \quad v_{i} \in V .
$$

If $V$ and $W$ are Banach spaces and $p(v)=\|v\|$, then we thus obtain on $\operatorname{Mult}^{n}(V, W)$ the structure of a Banach space. In this case we simply write $\|\beta\|$ for the norm of $\beta$ with respect to $p$.

(b) For $\beta \in \operatorname{Mult}^{n}(V, W)$, we write $\beta^{s}$ for the symmetrization of $\beta$ :

$$
\beta^{s}\left(x_{1}, \ldots, x_{n}\right):=\frac{1}{n !} \sum_{\sigma \in S_{n}} \beta\left(x_{\sigma(1)}, \ldots, x_{\sigma(n)}\right)
$$

and observe that $\left\|\beta^{s}\right\|_{p} \leq\|\beta\|_{p}$ for every $p \in \mathcal{P}(V)$.

(c) A map $f: V \rightarrow W$ is called homogeneous of degree $n$ if there exists a $\tilde{f} \in$ $\operatorname{Sym}^{n}(V, W)$ with

$$
f(x)=\tilde{f}(x, x, \ldots, x) \quad \text { for } \quad x \in V .
$$

Any such $f$ is smooth and $\tilde{f}$ can be recovered from $f$ by

$$
\widetilde{f}\left(v_{1}, \ldots, v_{n}\right)=\frac{1}{n !}\left(\partial_{v_{1}} \cdots \partial_{v_{n}} f\right)(0) .
$$

For $p \in \mathcal{P}(V)$, we define

$$
\|f\|_{p}:=\sup \{\|f(x)\|: p(x) \leq 1\} .
$$

Then we clearly have $\|f\|_{p} \leq\|\widetilde{f}\|_{p}$, and, in view of BS71a, Prop. 1.1], we also have

$$
\|\widetilde{f}\|_{p} \leq \frac{(2 n)^{n}}{n !}\|f\|_{p}
$$


Definition 3.2 Let $E$ be a Banach space.

(a) We call a linear map $\beta: U(\mathfrak{g}) \rightarrow E$ continuous if, for each $n \in \mathbb{N}_{0}$, the $n$-linear map

$$
\beta_{n}: \mathfrak{g}^{n} \rightarrow E, \quad\left(x_{1}, \ldots, x_{n}\right) \mapsto \beta\left(x_{1} \cdots x_{n}\right)
$$

is continuous and write $\operatorname{Hom}(U(\mathfrak{g}), E)_{c}$ for the set of all continuous linear maps. If $\mathfrak{g}$ is Banach-Lie algebra, we have on this space a family of seminorms

$$
p_{n}: \operatorname{Hom}(U(\mathfrak{g}), E)_{c} \rightarrow \mathbb{R}, \quad \beta \mapsto\left\|\beta_{n}\right\| .
$$

(b) We call $\beta \in \operatorname{Hom}(U(\mathfrak{g}), E)_{c}$ analytic if the series $\sum_{n=0}^{\infty} \frac{\beta\left(x^{n}\right)}{n !}$ converges on a 0 -neighborhood of $\mathfrak{g}$. The set of analytic elements is denoted $\operatorname{Hom}(U(\mathfrak{g}), E)_{\omega}$, and for $E=\mathbb{C}$ the elements of $\operatorname{Hom}(U(\mathfrak{g}), \mathbb{C})_{\omega}$ are called analytic functionals.

Lemma 3.3 (MNS09, Lemma 2.3]) If $\mathfrak{g}$ is a Banach-Lie algebra, then the sequence $\left(p_{n}\right)_{n \in \mathbb{N}_{0}}$ of seminorms turns the space $\operatorname{Hom}(U(\mathfrak{g}), E)_{c}$ into a Fréchet space for which all evaluation maps $\operatorname{ev}_{D}: \operatorname{Hom}(U(\mathfrak{g}), E)_{c} \rightarrow E, \beta \mapsto \beta(D)$ are continuous. The left regular representation of $U(\mathfrak{g})$ on this space defined by

$$
\mathfrak{g} \times \operatorname{Hom}(U(\mathfrak{g}), E)_{c} \rightarrow \operatorname{Hom}(U(\mathfrak{g}), E)_{c}, \quad(x, \beta) \mapsto-\beta \circ \lambda_{x}, \quad \lambda_{x}(D)=x D,
$$

and the right regular representation

$$
\mathfrak{g} \times \operatorname{Hom}(U(\mathfrak{g}), E)_{c} \rightarrow \operatorname{Hom}(U(\mathfrak{g}), E)_{c}, \quad(x, \beta) \mapsto \beta \circ \rho_{x}, \quad \rho_{x}(D)=D x,
$$

are continuous bilinear maps.

Proposition 3.4 If $\mathfrak{g}$ is a Baire space, f.i., if it is Fréchet, then an element $\beta \in$ $\operatorname{Hom}(U(\mathfrak{g}), E)_{c}$ is analytic if and only if there exists a $p \in \mathcal{P}(\mathfrak{g})$ with

$$
\sum_{n=0}^{\infty} \frac{\left\|\beta_{n}^{s}\right\|_{p}}{n !}<\infty
$$

If $\mathfrak{g}$ is Banach, this is equivalent to the existence of a $t>0$ with

$$
\sum_{n=0}^{\infty} \frac{\left\|\beta_{n}^{s}\right\| t^{n}}{n !}<\infty
$$

Proof. From (4) we obtain for $p(x)<1$ the estimate

$$
\sum_{n=0}^{\infty} \frac{\left\|\beta\left(x^{n}\right)\right\|}{n !}=\sum_{n=0}^{\infty} \frac{\left\|\beta_{n}^{s}(x, \ldots, x)\right\|}{n !} \leq \sum_{n=0}^{\infty} \frac{\left\|\beta_{n}^{s}\right\|_{p} p(x)^{n}}{n !} \leq \sum_{n=0}^{\infty} \frac{\left\|\beta_{n}^{s}\right\|_{p}}{n !}<\infty .
$$

Therefore (4) implies the uniform convergence of the series $\sum_{n} \frac{\beta\left(x^{n}\right)}{n !}$ for $p(x)<1$, hence in particular the analyticity of $\beta$. If $\mathfrak{g}$ is Banach, we obtain from (5) the uniform convergence for $\|x\|<t$.

Suppose, conversely, that $\beta$ is analytic. Then the functions $f_{n}(x):=\frac{\beta\left(x^{n}\right)}{n !}$ on $\mathfrak{g}$ are continuous and homogeneous of degree $n$. In view of [BS71b, Prop. 5.2], the convergence of the sequence $\sum_{n} f_{n}(x)$ on some 0 -neighborhood of $\mathfrak{g}$ implies the existence 
of some $q \in \mathcal{P}(\mathfrak{g})$ for which the series converges uniformly for $q(x) \leq 1$. In particular, there exists a $C>0$ with $\left\|\beta\left(x^{n}\right)\right\| \leq C n$ ! for $q(x) \leq 1$. We thus obtain with (3) the relation

$$
\left\|\beta_{n}^{s}\right\|_{q} \leq \frac{(2 n)^{n}}{n !}\left\|n ! f_{n}\right\|_{q} \leq n^{n} 2^{n}\left\|f_{n}\right\|_{q} \leq n^{n} 2^{n} C .
$$

Stierling's Formula

$$
n ! \approx \sqrt{2 \pi n}\left(\frac{n}{e}\right)^{n}
$$

leads to $\frac{n^{n}}{n !} \approx \frac{e^{n}}{\sqrt{2 \pi n}}$, so that we find with some $C^{\prime}>0$

$$
\sum_{n=0}^{\infty} \frac{\left\|\beta_{n}^{s}\right\|_{q} t^{n}}{n !} \leq C^{\prime} \sum_{n=0}^{\infty} \frac{e^{n}}{\sqrt{n}}(2 t)^{n}=C^{\prime} \sum_{n=0}^{\infty} \frac{(2 e t)^{n}}{\sqrt{n}} .
$$

This series converges for $t<\frac{1}{2 e}$, so that the assertion follows for any such $t$ with $p:=t^{-1} q$.

Definition 3.5 If $\beta \in \operatorname{Hom}(U(\mathfrak{g}), E)_{c}$ is analytic and $\sum_{n=0}^{\infty} \frac{\left\|\beta_{n}^{s}\right\|_{p} t^{n}}{n !}<\infty$ for some $t>0$, then

$$
\sum_{n=0}^{\infty} \frac{\left\|\beta\left(x^{n}\right)\right\|}{n !} \leq \sum_{n=0}^{\infty} \frac{\left\|\beta_{n}^{s}\right\|_{p} p(x)^{n}}{n !}<\infty
$$

holds for $p(x)<t$ and

$$
\left.\left.R_{\beta, p}:=\sup \left\{t>0: \sum_{n=0}^{\infty} \frac{\left\|\beta_{n}^{s}\right\|_{p} t^{n}}{n !}<\infty\right\} \in\right] 0, \infty\right]
$$

is called the $p$-radius of convergence of $\beta$. If $R_{\beta, p}<\infty$, we say that $\beta$ is $p$-analytic, or, analytic with respect to $p$. According to Hadamard's Formula, we have

$$
R_{\beta, p}^{-1}=\limsup \sqrt[n]{\frac{\left\|\beta_{n}^{s}\right\|_{p}}{n !}}
$$

Note that, for each $r>0$, the set $\operatorname{Hom}(U(\mathfrak{g}), E)_{\omega, p, r}$ of analytic maps $\beta$ with $R_{\beta, p} \geq r$ is a linear subspace. We write $\operatorname{Hom}(U(\mathfrak{g}), E)_{\omega, p}$ for the union of all these spaces, which also is a linear subspace.

We now come to the main result of this section.

Theorem 3.6 If $p \in \mathcal{P}(\mathfrak{g})$ is submultiplicative and $r>0$, then $\operatorname{Hom}(U(\mathfrak{g}), E)_{\omega, p, r}$ is invariant under the left and right regular action of $\mathfrak{g}$. In particular, the space $\operatorname{Hom}(U(\mathfrak{g}), E)_{\omega, p}$ of analytic elements is invariant.

Proof. For $\beta \in \operatorname{Hom}(U(\mathfrak{g}), E)_{\omega, p, r}, y \in \mathfrak{g}, n \in \mathbb{N}_{0}$, and $1 \leq k \leq n+1$, we define

$$
\left(i_{y}^{k} \beta\right)_{n} \in \operatorname{Mult}^{n}(\mathfrak{g}, E), \quad\left(i_{y}^{k} \beta\right)_{n}\left(x_{1}, \ldots, x_{n}\right):=\beta\left(x_{1} \cdots x_{k-1} y x_{k} \cdots x_{n}\right) .
$$


For $n \in \mathbb{N}_{0}$ and $y \in \mathfrak{g}$ we put

$$
c_{n}:=\inf \left\{C>0:(\forall y \in \mathfrak{g})(\forall k \leq n+1)\left\|\left(i_{y}^{k} \beta\right)_{n}^{s}\right\|_{p} \leq C p(y)\right\} .
$$

Now let $y \in \mathfrak{g}$. Our first goal is an estimate for the numbers $c_{n}$. For $n=0$ we have $k=1$ and $i_{y}^{1} \beta=\beta(y)$, so that

$$
c_{0}=\left\|\beta_{1}\right\|_{p}=\left\|\beta_{1}^{s}\right\|_{p} .
$$

Our strategy is to compare the two symmetric $n$-linear maps $\left(i_{y}^{k} \beta\right)_{n}^{s}$ and $i_{y}\left(\beta_{n+1}^{s}\right)=$ $\beta_{n+1}^{s}(y, \cdots)$ :

$$
\begin{aligned}
& \left(i_{y}^{k} \beta\right)_{n}^{s}\left(x_{1}, \ldots, x_{n}\right)-\beta_{n+1}^{s}\left(y, x_{1}, \ldots, x_{n}\right) \\
& =\frac{1}{n !}\left(\sum_{\sigma \in S_{n}} \beta\left(x_{\sigma(1)} \cdots x_{\sigma(k-1)} y x_{\sigma(k)} \cdots x_{\sigma(n)}\right)\right. \\
& \left.\quad-\frac{1}{n+1} \sum_{j=1}^{n+1} \sum_{\sigma \in S_{n}} \beta\left(x_{\sigma(1)} \cdots x_{\sigma(j-1)} y x_{\sigma(j)} \cdots x_{\sigma(n)}\right)\right) \\
& =\frac{1}{(n+1) !} \sum_{j=1}^{n+1} \sum_{\sigma \in S_{n}} \\
& \quad\left(\beta\left(x_{\sigma(1)} \cdots x_{\sigma(k-1)} y x_{\sigma(k)} \cdots x_{\sigma(n)}\right)-\beta\left(x_{\sigma(1)} \cdots x_{\sigma(j-1)} y x_{\sigma(j)} \cdots x_{\sigma(n)}\right)\right) .
\end{aligned}
$$

With the relation $\left[y, x_{1} \cdots x_{a}\right]=\sum_{j=1}^{a} x_{1} \cdots x_{j-1}\left[y, x_{j}\right] x_{j+1} \cdots x_{a}$ we obtain for $k<j$

$$
\begin{aligned}
& \beta\left(x_{\sigma(1)} \cdots x_{\sigma(k-1)} y x_{\sigma(k)} \cdots x_{\sigma(n)}\right)-\beta\left(x_{\sigma(1)} \cdots x_{\sigma(j-1)} y x_{\sigma(j)} \cdots x_{\sigma(n)}\right) \\
& =\beta\left(x_{\sigma(1)} \cdots x_{\sigma(k-1)}\left[y, x_{\sigma(k)} \cdots x_{\sigma(j-1)}\right] x_{\sigma(j)} \cdots x_{\sigma(n)}\right) \\
& =\sum_{\ell=k}^{j-1} \beta\left(x_{\sigma(1)} \cdots x_{\sigma(\ell-1)}\left[y, x_{\sigma(\ell)}\right] x_{\sigma(\ell+1)} \cdots x_{\sigma(n)}\right) .
\end{aligned}
$$

Next we observe that

$$
\begin{aligned}
& \sum_{\sigma \in S_{n}} \beta\left(x_{\sigma(1)} \cdots x_{\sigma(\ell-1)}\left[y, x_{\sigma(\ell)}\right] x_{\sigma(\ell+1)} \cdots x_{\sigma(n)}\right) \\
& =(n-1) ! \sum_{m=1}^{n}\left(i_{\left[y, x_{m}\right]}^{\ell} \beta\right)_{n-1}^{s}\left(x_{1}, \ldots, \widehat{x_{m}}, \ldots, x_{n}\right),
\end{aligned}
$$

where $\widehat{x_{m}}$ denotes omission of $x_{m}$. The norm of this sum can be estimated by

$$
\begin{aligned}
& (n-1) ! \sum_{m=1}^{n} c_{n-1} p\left(\left[y, x_{m}\right]\right) p\left(x_{1}\right) \cdots p\left(\widehat{x}_{m}\right) \cdots p\left(x_{n}\right) \\
& \leq(n-1) ! \sum_{m=1}^{n} c_{n-1} p(y) p\left(x_{1}\right) \cdots p\left(x_{n}\right)=n ! c_{n-1} p(y) p\left(x_{1}\right) \cdots p\left(x_{n}\right) .
\end{aligned}
$$


For $k>j$ we likewise obtain

$$
\begin{aligned}
& \beta\left(x_{\sigma(1)} \cdots x_{\sigma(k-1)} y x_{\sigma(k)} \cdots x_{\sigma(n)}\right)-\beta\left(x_{\sigma(1)} \cdots x_{\sigma(j-1)} y x_{\sigma(j)} \cdots x_{\sigma(n)}\right) \\
& =\beta\left(x_{\sigma(1)} \cdots x_{\sigma(j-1)}\left[x_{\sigma(j)} \cdots x_{\sigma(k-1)}, y\right] x_{\sigma(k)} \cdots x_{\sigma(n)}\right) \\
& =\sum_{\ell=j}^{k-1} \beta\left(x_{\sigma(1)} \cdots x_{\sigma(\ell-1)}\left[x_{\sigma(\ell)}, y\right] x_{\sigma(\ell+1)} \cdots x_{\sigma(n)}\right)
\end{aligned}
$$

which can be estimated in the same way. This leads to the estimate

$$
\begin{aligned}
& \left\|\left(i_{y}^{k} \beta\right)_{n}^{s}\left(x_{1}, \ldots, x_{n}\right)-\beta_{n+1}^{s}\left(y, x_{1}, \ldots, x_{n}\right)\right\| \\
& \leq \frac{1}{(n+1) !}\left(\sum_{j>k} \sum_{\ell=k}^{j-1} n ! c_{n-1} p(y) p\left(x_{1}\right) \cdots p\left(x_{n}\right)+\sum_{j<k} \sum_{\ell=j}^{k-1} n ! c_{n-1} p(y) p\left(x_{1}\right) \cdots p\left(x_{n}\right)\right) \\
& \leq \frac{1}{n+1} \sum_{j \neq k}|k-j| c_{n-1} p(y) p\left(x_{1}\right) \cdots p\left(x_{n}\right) \leq \frac{n^{2}}{n+1} c_{n-1} p(y) p\left(x_{1}\right) \cdots p\left(x_{n}\right) \\
& \leq n c_{n-1} p(y) p\left(x_{1}\right) \cdots p\left(x_{n}\right) .
\end{aligned}
$$

We conclude that

$$
\left\|\left(i_{y}^{k} \beta\right)_{n}^{s}\left(x_{1}, \ldots, x_{n}\right)\right\| \leq\left\|\beta_{n+1}^{s}\right\|_{p} p(y) p\left(x_{1}\right) \cdots p\left(x_{n}\right)+n c_{n-1} p(y) p\left(x_{1}\right) \cdots p\left(x_{n}\right),
$$

i.e.,

$$
c_{n} \leq\left\|\beta_{n+1}^{s}\right\|_{p}+n c_{n-1} .
$$

Iterating this estimate leads to

$$
\begin{aligned}
c_{n} \leq\left\|\beta_{n+1}^{s}\right\|_{p}+n\left\|\beta_{n}^{s}\right\|_{p}+n(n-1)\left\|\beta_{n-1}^{s}\right\|_{p} \\
+\cdots+n(n-1) \cdots 2 \cdot\left\|\beta_{2}^{s}\right\|_{p}+n !\left\|\beta_{1}^{s}\right\|_{p} .
\end{aligned}
$$

For $0<t<\min \left(1, R_{\beta, p}\right)$, there exists a $C>0$ with

$$
\left\|\beta_{n}^{s}\right\|_{p} \leq C n ! t^{-n} \quad \text { for } \quad n \in \mathbb{N} .
$$

We thus obtain

$$
\begin{aligned}
c_{n} & \leq C\left((n+1) ! t^{-n-1}+n ! n t^{-n}+\cdots+n \cdots 2 \cdot 2 ! t^{-2}+n ! t^{-1}\right) \\
& \leq C(n+1) \cdot(n+1) ! t^{-n-1} \leq C(n+2) ! t^{-n-1} .
\end{aligned}
$$

This implies that

$$
\sum_{n} \frac{c_{n} s^{n}}{n !} \leq C \sum_{n}(n+2)(n+1) t^{-1}(s / t)^{n}
$$

which converges for $s<t$.

Finally we observe that $\left(\beta \circ \rho_{y}\right)_{n}^{s}=\left(i_{y}^{n+1} \beta\right)_{n}^{s}$, so that

$$
\left\|\left(\beta \circ \rho_{y}\right)_{n}^{s}\right\|_{p}=\left\|\left(i_{y}^{n+1} \beta\right)_{n}^{s}\right\|_{p} \leq c_{n} p(y)
$$

shows that $\beta \circ \rho_{y}$ is analytic with $R_{\beta \circ \rho_{y}, p} \geq R_{\beta, p}$. Similarly $\left(\beta \circ \lambda_{y}\right)_{n}^{s}=\left(i_{y}^{1} \beta\right)_{n}^{s}$ implies that $\beta \circ \lambda_{y}$ is analytic with $R_{\beta \circ \lambda_{y}, p} \geq R_{\beta, p}$. 
Remark 3.7 It is an interesting question whether (for Banach-Lie algebras) the condition $\sum_{n=0}^{\infty} \frac{\left\|\beta_{n}^{s}\right\| t^{n}}{n !}<\infty$ characterizing analytic elements in $\operatorname{Hom}(U(\mathfrak{g}), E)_{c}$ also implies the stronger condition $\sum_{n=0}^{\infty} \frac{\left\|\beta_{n}\right\| t^{n}}{n !}<\infty$ on the non-symmetrized maps $\beta_{n}$. A natural way to verify this would be to compare the norms of $\beta_{n}$ and $\beta_{n}^{s}$. For $x_{1}, \ldots, x_{n} \in \mathfrak{g}$ we have

$$
\left(\beta_{n}-\beta_{n}^{s}\right)\left(x_{1}, \ldots, x_{n}\right)=\frac{1}{n !} \sum_{\sigma \in S_{n}} \beta\left(x_{1} \cdots x_{n}\right)-\beta\left(x_{\sigma(1)} \cdots x_{\sigma(n)}\right) .
$$

Writing a permutation in terms of transpositions $s_{j}$ exchanging $j$ and $j+1$ and using that the corresponding word length satisfies $\ell(\sigma) \leq \frac{n(n-1)}{2}$, we obtain as in the proof of Theorem 3.6 an estimate of the form

$$
\left\|\beta_{n}-\beta_{n}^{s}\right\| \leq \frac{n(n-1)}{2}\left\|\beta_{n-1}\right\|
$$

which leads to the recursive estimate

$$
\left\|\beta_{n}\right\| \leq\left\|\beta_{n}^{s}\right\|+\frac{n(n-1)}{2}\left\|\beta_{n-1}\right\| .
$$

Iteration now yields

$$
\begin{aligned}
& \left\|\beta_{n}\right\| \\
& \leq\left\|\beta_{n}^{s}\right\|+\frac{n(n-1)}{2}\left\|\beta_{n-1}^{s}\right\|+\frac{n(n-1)^{2}(n-2)}{2^{2}}\left\|\beta_{n-2}^{s}\right\|+\cdots+\frac{\left.n(n-1)^{2} \cdots 2^{2} \cdot 1\right)}{2^{n-1}}\left\|\beta_{1}\right\| \\
& \leq \sum_{k=0}^{n-1} \frac{(n !)^{2}}{((n-k) !)^{2} 2^{k}}\left\|\beta_{n-k}^{s}\right\| .
\end{aligned}
$$

From the estimate $\left\|\beta_{n}^{s}\right\| \leq C n ! t^{-n}$, we thus obtain

$$
\begin{aligned}
\sum_{n} \frac{\left\|\beta_{n}\right\| t^{n}}{n !} & \leq C \sum_{n} \frac{t^{n}}{n !} \sum_{k=0}^{n-1} \frac{(n !)^{2}}{((n-k) !)^{2} 2^{k}} t^{k-n}(n-k) ! \\
& \leq C \sum_{n} \sum_{k=0}^{n-1} \frac{n !}{(n-k) !}(t / 2)^{k}=C \sum_{k}(t / 2)^{k} \sum_{n>k} \frac{n !}{(n-k) !}=\infty .
\end{aligned}
$$

Apparently this estimate does not lead to the desired convergence, and we do not know if the series $\sum_{n} \frac{\left\|\beta_{n}\right\| t^{n}}{n !}$ converges for some $t>0$.

For finite dimensional Lie algebras, analytic vectors are usually defined by a condition equivalent to the convergence of the series $\sum_{n} \frac{\left\|\beta_{n}\right\| t^{n}}{n !}$ for some $t>0$ (cf. Sch90, Sect. 10.4]). At first sight this is stronger than the present one (cf. Sch90, Lemma 10.49]), but according to Nel59, Lemma 7.1, Thm. 2] it is equivalent. We do not know if this is also true for Banach-Lie algebras.

Remark 3.8 (a) If the topology on $\mathfrak{g}$ is defined by a set of submultiplicative seminorms, then Theorem 3.6 implies the invariance of the space $\operatorname{Hom}(U(\mathfrak{g}), E)_{\omega}$ of analytic elements under the left and right regular action. 
(b) We do not know if this is true for general locally convex Lie algebras. Here the critical point is that, if $c_{n}^{p}$ denotes the constant $c_{n}$, defined with respect to the seminorm $p$, and $p([x, y]) \leq q(x) q(y)$ for some $q \in \mathcal{P}(\mathfrak{g})$ with $q \geq p$, then the argument in the proof of Theorem 3.6 only yields an estimate of the form

$$
c_{n}^{q} \leq\left\|\beta_{n+1}^{s}\right\|_{p}+n c_{n-1}^{p} .
$$

We do not see how to use this estimate to conclude the invariance of $\operatorname{Hom}(U(\mathfrak{g}), E)_{\omega}$.

\section{Analytic vectors and continuity of Lie algebra rep- resentations}

In this section $\mathbb{K} \in\{\mathbb{R}, \mathbb{C}\}$ and all vector spaces are $\mathbb{K}$-vector spaces. Before we turn to the connection between analytic functionals and unitary representations of Lie groups, we have to clarify some continuity requirements for representations of topological Lie algebras on topological vector spaces.

\section{Continuity of Lie algebra representations}

Definition 4.1 Let $(\rho, V)$ be a representation of the topological Lie algebra $\mathfrak{g}$ on the topological vector space $V$.

(a) We say that $\rho$ is strongly continuous if for every $v \in V$ the map

$$
\beta_{v}: \mathfrak{g} \rightarrow V, \quad x \mapsto \rho(x) v
$$

is continuous. For a finite dimensional Lie algebra $\mathfrak{g}$, this condition is always satisfied.

(b) If $E \subseteq V^{\prime}$ is a subspace separating the points of the completion $\widehat{V}$ of $V$, then $\rho$ is called weakly continuous with respect to $E$ if, for $\alpha \in E$ and $v \in V$, the linear functional $x \mapsto \alpha(\rho(x) v)$ is continuous. 2

(c) If $V$ is a complex pre-Hilbert space, then we call $\rho$

(i) weakly continuous if, for any two vectors $v, w \in V$, the map $\mathfrak{g} \rightarrow \mathbb{C}, x \mapsto\langle\rho(x) v, w\rangle$ is continuous, i.e., if $\rho$ is weakly continuous with respect to $E=\{\langle\cdot, v\rangle: v \in V\}$.

(ii) a $*$-representation if

$$
\langle\rho(x) v, w\rangle=-\langle v, \rho(x) w\rangle \quad \text { for } \quad x \in \mathfrak{g}, v, w \in V .
$$

Lemma 4.2 If $\mathfrak{g}$ is a Fréchet space and $V$ is a metrizable locally convex space, then every weakly continuous representation is strongly continuous.

\footnotetext{
${ }^{2}$ If $V$ is a normed space which is not complete and $\widehat{v} \in \widehat{V} \backslash V$, then its annihilator $E:=\widehat{v}^{\perp} \subseteq$ $V^{\prime} \cong \widehat{V}^{\prime}$ is a hyperplane with $E^{\perp} \cap V=\mathbb{K} \widehat{v} \cap V=\{0\}$. Therefore $E$ separates the points of $V$ but not of $\widehat{V}$.
} 
Proof. We claim that the graph of $\beta_{v}$, considered as a linear map into the Fréchet completion $\widehat{V}$ of $V$, is closed. Since every continuous linear functional on $V$ extends uniquely to a continuous linear functional on the completion $\widehat{V}$, we identify the two spaces $\widehat{V}^{\prime}$ and $V^{\prime}$.

Suppose that $x_{n} \rightarrow x$ in $\mathfrak{g}$ and $\beta_{v}\left(x_{n}\right) \rightarrow w \in \widehat{V}$ and let $E \subseteq \widehat{V}^{\prime}$ be a subspace separating the points of $\widehat{V}$ such that all linear functionals $\mathfrak{g} \rightarrow \mathbb{K}, x \mapsto \alpha(\rho(x) v), \alpha \in E$, $v \in V$, are continuous. Then $\alpha\left(\rho\left(x_{n}\right) v\right) \rightarrow \alpha(\rho(x) v)$ and $\alpha\left(\rho\left(x_{n}\right) v\right) \rightarrow \alpha(w)$ implies $w=\rho(x) v$. Therefore the graph of $\beta_{v}$ is closed, so that the Closed Graph Theorem ([Bou07, Ch. 1, $\S 3$, Cor. 5]) implies that $\beta_{v}$ is continuous.

The following proposition is classical for $k=2$, but here we need a more general version.

Proposition 4.3 If $F_{1}, \ldots, F_{k}$ are Fréchet spaces, $V$ a topological vector space, and $\beta: F_{1} \times \cdots \times F_{k} \rightarrow V$ a k-linear map which is separately continuous, then $\beta$ is continuous.

Proof. We argue by induction over $k$. The case $k=1$ is trivial, so that we may assume that $k>1$. Let $F:=F_{1} \times \cdots \times F_{k-1}$ and consider the map

$$
\Omega: F \times F_{k} \rightarrow V, \quad(x, y)=\left(\left(x_{1}, \ldots, x_{k-1}\right), y\right) \mapsto \beta\left(x_{1}, \ldots, x_{k-1}, y\right) .
$$

For $y \in F_{k}$, our induction hypothesis implies that the $(k-1)$-linear map $\Omega(\cdot, y): F \rightarrow V$ is continuous, so that the continuity of $\Omega$ follows from [Ne10, Prop. 5.1].

Lemma 4.4 If the representation $(\rho, V)$ is strongly continuous, then, for each $n \in \mathbb{N}$ and every $v \in V$, the $n$-linear map

$$
\beta_{v}^{n}: \mathfrak{g}^{n} \rightarrow V, \quad\left(x_{1}, \ldots, x_{n}\right) \mapsto \rho\left(x_{1}\right) \cdots \rho\left(x_{n}\right) v
$$

is separately continuous. If, in addition, $\mathfrak{g}$ is Fréchet, then each $\beta_{v}^{n}$ is continuous.

Proof. We argue by induction. By definition, the assertion holds for $n=1$. We assume that each map $\beta_{w}^{n-1}$ is continuous in each argument. For $j<n$, the continuity of $\beta_{v}^{n}$ in $x_{j}$ then follows from the continuity of $\beta_{\rho\left(x_{n}\right) v}^{n-1}$ in each argument. Next we observe that

$$
\begin{aligned}
\beta_{v}^{n}\left(x_{1}, \ldots, x_{n}\right) & =\beta_{v}^{n}\left(x_{1}, \ldots, x_{n-2}, x_{n}, x_{n-1}\right)+\rho\left(x_{1}\right) \cdots \rho\left(x_{n-2}\right) \rho\left(\left[x_{n-1}, x_{n}\right]\right) v \\
& =\beta_{v}^{n}\left(x_{1}, \ldots, x_{n-2}, x_{n}, x_{n-1}\right)+\beta_{v}^{n-1}\left(x_{1}, \ldots, x_{n-2},\left[x_{n-1}, x_{n}\right]\right) .
\end{aligned}
$$

From the continuity of the Lie bracket on $\mathfrak{g}$ it now follows that both terms on the right are continuous in $x_{n}$. This proves the continuity of $\beta_{v}^{n}$ in $x_{n}$.

Now $\beta_{v}^{n}: \mathfrak{g} \rightarrow V$ is a separately continuous multilinear map, hence continuous if $\mathfrak{g}$ is a Fréchet space (Proposition 4.3). 
Lemma 4.5 Suppose that $V$ is a pre-Hilbert space. Then a $*$-representation $(\rho, V)$ of the Fréchet-Lie algebra $\mathfrak{g}$ on $V$ is strongly continuous if and only if for each $v \in V$ the quadratic map $q_{v}: \mathfrak{g} \rightarrow \mathbb{R}, x \mapsto\left\langle\rho(x)^{2} v, v\right\rangle$ is continuous.

Proof. If each map $q_{v}$ is continuous, then $\|\rho(x) v\|^{2}=-q_{v}(x)$ implies that $\rho$ is strongly continuous.

If, conversely, $\rho$ is strongly continuous, then Lemma 4.4 implies that the bilinear maps $\beta_{v}^{2}: \mathfrak{g}^{2} \rightarrow V,(x, y) \mapsto \rho(x) \rho(y) v$ are continuous, and this implies the continuity of the maps $q_{v}$.

Proposition 4.6 If $(\pi, V)$ is a continuous representation of the Lie group $G$ on $V$, then the derived representation $\mathrm{d} \pi$ of $\mathfrak{g}$ on the space $V^{\infty}$ of smooth vectors is strongly continuous with respect to the subspace topology induced from $V$.

Proof. If $v$ is a smooth vector and $\pi^{v}: G \rightarrow V, g \mapsto \pi(g) v$, its smooth orbit map, then $x \mapsto \mathrm{d} \pi(x) v=T_{(\mathbf{1}, 0)}\left(\pi^{v}\right)(x, 0)$ is a continuous linear map.

In [Ne10, Thm. 8.2] we show that the preceding observation can even be sharpened if $G$ and $V$ are Banach. If $\mathcal{D}_{\mathfrak{g}} \subseteq V$ denotes the common domain of the generators $\overline{\mathrm{d} \pi(x)}$ of the one-parameter groups $t \mapsto \pi(\exp t x), x \in \mathfrak{g}$, then, for each $v \in \mathcal{D}_{\mathfrak{g}}$, the map $\beta_{v}: \mathfrak{g} \rightarrow V$ is continuous and linear. In this sense the continuity requirement is satisfied for any representation of a Banach-Lie algebra that comes from a continuous Lie group action on a Banach space.

\section{Analytic vectors}

We are now ready to turn to the central concept of this article.

Definition 4.7 Let $A$ be an operator with domain $\mathcal{D}(A)$ on the Banach space $E$. An element in the space $\mathcal{D}^{\infty}(A):=\bigcap_{n=1}^{\infty} \mathcal{D}\left(A^{n}\right)$ is called a smooth vector for $A$, and a smooth vector is said to be analytic if there exists a $t>0$ with

$$
\sum_{n=0}^{\infty} \frac{\left\|A^{n} v\right\| t^{n}}{n !}<\infty .
$$

We write $\mathcal{D}^{\omega}(A)$ for the space of analytic vectors for $A$.

An important application of the concept of an analytic vector is that it provides the following criterion for essential selfadjointness:

Theorem 4.8 (Nelson's Theorem; [Nel59; [RS75, Thm. X.39]) A symmetric operator $A$ on a Hilbert space $\mathcal{H}$ for which the space $\mathcal{D}^{\omega}(A)$ of analytic vectors is dense is essentially selfadjoint.

Definition 4.9 Let $(\rho, V)$ be a strongly continuous representation of $\mathfrak{g}$ on the normed space $V$. Then an element $v \in V$ is called an analytic vector if $\sum_{n=0}^{\infty} \frac{\left\|\rho(x)^{n} v\right\|}{n !}$ converges for every $x$ in a 0 -neighborhood of $\mathfrak{g}$. 
Proposition 4.10 (Automatic simultaneous analyticity) Let $(\rho, V)$ be a strongly continuous representation of the Lie algebra $\mathfrak{g}$ on the normed space $V$. If $\mathfrak{g}$ is a Baire space (which is in particular the case if $\mathfrak{g}$ is Fréchet), then every $v \in V$ which is an analytic vector for all the operators $\rho(x), x \in \mathfrak{g}$, is an analytic vector.

Proof. In view of Lemma 4.4, the functions $f_{n}(x):=\frac{\rho(x)^{n} v}{n !}$ are continuous and homogeneous of degree $n$. The assumption that $v$ is analytic for every operator $\rho(x)$ implies that the set $S$ of all elements $x \in \mathfrak{g}$ for which the series $\sum_{n} f_{n}(x)$ converges in the completion $\widehat{V}$ of $V$ is absorbing, i.e., $\bigcup_{n \in \mathbb{N}} n \cdot S=\mathfrak{g}$. Now [BS71b, Prop. 5.2(1)] implies the uniform convergence of this series on a 0 -neighborhood of $\mathfrak{g}$, i.e., $v$ is an analytic vector.

\section{$5 \quad$ Analytic vectors of unitary representations}

The main result of this section asserts that a vector $v$ in a Hilbert space $\mathcal{H}$ is analytic with respect to a continuous unitary representation $(\pi, \mathcal{H})$ of an analytic Lie group $G$ if and only if the matrix coefficient $\pi^{v, v}(g)=\langle\pi(g) v, v\rangle$ is analytic in a 1-neighborhood. This will be a direct consequence of the following theorem characterizing analyticity of $\mathcal{H}$-valued maps in terms of analyticity of the corresponding kernel. The case of this theorem, where $M$ is one-dimensional, can already be found in [Kr49, p. 81].

Theorem 5.1 Let $M$ be an analytic Fréchet manifold and $\mathcal{H}$ be a Hilbert space. Then a function $\gamma: M \rightarrow \mathcal{H}$ is analytic if and only if the kernel $K(x, y):=\langle\gamma(x), \gamma(y)\rangle$ is analytic on an open neighborhood of the diagonal in $M \times M$.

Proof. Step 1: We assume w.l.o.g. that $\mathcal{H}$ is a real Hilbert space. Since compositions of analytic maps are analytic ([G102, Prop. 2.8]), the analyticity of $\gamma$ implies the analyticity of the kernel $K$. Suppose, conversely, that $K$ is analytic. As our assertion is local, we may assume that $M$ is an open subset of a locally convex space $V$ containing 0 , that $K$ is analytic on $M \times M$, and that we have to show the analyticity of $\gamma$ in a 0 -neighborhood. To this end, we may further assume that $U$ is a convex symmetric 0-neighborhood for which $K$ extends to a holomorphic function on $W:=(U+i U) \times(U+i U) \subseteq V_{\mathbb{C}} \times V_{\mathbb{C}}$. Shrinking $W$ if necessary, we may further assume that

$$
C:=\sup \{|K(z, w)|:(z, w) \in W\}<\infty .
$$

[Ne10, Thm 7.1] implies that $\gamma$ is smooth.

Step 2: Next we consider the special case $\mathcal{H}=\mathbb{R}$. Since $K$ is analytic, it is in particular smooth, so that $\gamma$ is a smooth curve in $\mathcal{H}$, and

$$
\left\|\gamma^{(n)}(t)\right\|^{2}=\left.\frac{d^{n}}{d t^{n}} \frac{d^{n}}{d s^{n}}\langle\gamma(t), \gamma(s)\rangle\right|_{t=s}=\left(\partial_{1}^{n} \partial_{2}^{n} K\right)(t, t) .
$$

For any $t \in U$ and $r>0$ for which

$$
W_{r}(t):=\left\{z \in \mathbb{C}^{2}:\left|z_{1}-t\right|,\left|z_{2}-t\right| \leq r\right\} \subseteq W
$$


we have the Cauchy estimates

$$
\left|\left(\partial_{1}^{n} \partial_{2}^{m} K\right)(t, t)\right| \leq C n ! m ! r^{-n-m} .
$$

We conclude that

$$
\left\|\gamma^{(n)}(t)\right\| \leq \sqrt{C} n ! r^{-n} .
$$

Step 3: For a general $\mathcal{H}$, we consider for $x \in U$ and $h \in V$ the curves

$$
\gamma_{x, h}(t):=\gamma(x+t h)
$$

which are analytic in a neighborhood of $t=0$ by Step 2. To prove the convergence of the Taylor series of $\gamma$ in a 0-neighborhood, we observe that

$$
\gamma_{x, h}^{(n)}(0)=\left(\mathrm{d}^{n} \gamma\right)(x)(h, \ldots, h),
$$

so that (8) implies that

$$
\left\|\left(\mathrm{d}^{n} \gamma\right)(x)(h, \ldots, h)\right\|=\left\|\gamma_{x, h}^{(n)}(0)\right\| \leq n ! 2^{-n} \sqrt{C}
$$

whenever $\left(x+z_{1} h, x+z_{2} h\right) \in W$ holds for $\left|z_{i}\right| \leq 2$. We conclude that the Taylor series

$$
\sum_{n=0}^{\infty} \frac{1}{n !}\left(\mathrm{d}^{n} \gamma\right)(x)(h, \ldots, h)
$$

of $\gamma$ in $x$ converges uniformly for all pairs $(x, h)$ in an open neighborhood of $(0,0)$. Since the remainder term in the Taylor expansion satisfies

$$
\begin{aligned}
& \left\|\gamma(x+h)-\sum_{k=0}^{n} \frac{1}{n !}\left(\mathrm{d}^{n} \gamma\right)(x)(h, \ldots, h)\right\| \\
& \leq \frac{1}{(n+1) !} \int_{0}^{1}\left\|\left(\mathrm{~d}^{n+1} f\right)(x+t h)(h, \ldots, h)\right\| d t \leq 2^{-n-1} \sqrt{C} \rightarrow 0,
\end{aligned}
$$

the Taylor series actually converges to $\gamma(x+h)$ for $x, h$ close to 0 . In view of BS71b, Thm. 7.2], this implies that $\gamma$ is analytic in the sense defined above.

The following theorem is an analytic version of [Ne10, Thm. 7.2], which is concerned with smooth vectors (cf. Go69, Prop. 4.1] for the case $G=\mathbb{R}$ ).

Theorem 5.2 If $(\pi, \mathcal{H})$ is a unitary representation of an analytic Fréchet-Lie group $G$, then $v \in \mathcal{H}$ is an analytic vector if and only if the corresponding matrix coefficient $\pi^{v, v}(g):=\langle\pi(g) v, v\rangle$ is analytic on a 1-neighborhood in $G$.

Proof. (cf. [Ne10, Thm. 7.2] for smooth vectors) Clearly, $\pi^{v, v}$ is analytic for $v \in \mathcal{H}^{\omega}$. Suppose, conversely, that $\pi^{v, v}$ is analytic in a 1-neighborhood $U \subseteq G$ and that $U^{\prime}$ is a 1-neighborhood with $h^{-1} g \in U$ for $g, h \in U^{\prime}$. In view of Theorem 5.1, the analyticity of $\pi^{v}(g)=\pi(g) v$ on $x U^{\prime}, x \in G$, is equivalent to the analyticity of the function

$$
(g, h) \mapsto\langle\pi(x g) v, \pi(x h) v\rangle=\pi^{v, v}\left(h^{-1} g\right)
$$

on $U^{\prime} \times U^{\prime}$, which follows from the analyticity of $\pi^{v, v}$ on $U$. 
In view of the GNS construction, we obtain an analog of [Ne10, Cor. 7.4] for analytic functions:

Corollary 5.3 If $\varphi$ is a positive definite function on a Lie group $G$ which is analytic in a 1-neighborhood, then $\varphi$ is analytic.

Problem 5.4 Suppose that $(\pi, V)$ is a smooth representation of the Banach-Lie group $G$ on the Banach space $V$. Is the space $V^{\omega}$ of analytic vectors dense? This is true if $\operatorname{dim} G<\infty$ for any continuous Banach representation ([Nel59, Thm. 4], Ga60]), but the proof is based on the integrated representation to $L^{1}(G)$ and mollifying with the heat kernel. All these techniques are not available for general infinite dimensional groups. However, some Lie groups carry so-called heat kernel measures which behave in many respects like Haar measure with a heat kernel density (cf. [DG08).

\section{Positive analytic functionals}

In this section we turn to the $*$-aspects of analytic functionals on $U(\mathfrak{g})$. Throughout $U_{\mathbb{C}}(\mathfrak{g}):=U\left(\mathfrak{g}_{\mathbb{C}}\right) \cong U(\mathfrak{g})_{\mathbb{C}}$ denotes the enveloping algebra of the complexified Lie algebra $\mathfrak{g}_{\mathbb{C}}$. It coincides with the complexification of the real algebra $U(\mathfrak{g})$ and we have $\operatorname{Hom}_{\mathbb{R}}(U(\mathfrak{g}), E) \cong \operatorname{Hom}_{\mathbb{C}}\left(U_{\mathbb{C}}(\mathfrak{g}), E\right)$ for every complex vector space $E$.

Definition 6.1 We write $*$ for the unique antilinear antiautomorphism of $U_{\mathbb{C}}(\mathfrak{g})$ with $x^{*}=-x$ for $x \in \mathfrak{g}$. Then $\left(U_{\mathbb{C}}(\mathfrak{g}), *\right)$ is an involutive algebra and a linear functional $\lambda \in U_{\mathbb{C}}(\mathfrak{g})^{*}$ is called positive if $\lambda\left(D^{*} D\right) \geq 0$ for $D \in U_{\mathbb{C}}(\mathfrak{g})$.

Typical examples of positive functional arise from $*$-representations $(\rho, \mathcal{D})$ on preHilbert spaces (see Definition 4.1(b)(ii)) as $\lambda(D)=\langle\rho(D) v, v\rangle$, and a suitable variant of the GNS construction implies that every positive functional on $U_{\mathbb{C}}(\mathfrak{g})$ is of this from ([Po71, Thm. 6.3], [Sch90, Thm. 8.6.2]).

Definition 6.2 A positive linear functional $\lambda \in U_{\mathbb{C}}(\mathfrak{g})^{*}$ is said to be integrable if there exists a smooth positive definite function $\varphi: G \rightarrow \mathbb{C}$ with

$$
\lambda(D)=(D \varphi)(\mathbf{1}) \quad \text { for } \quad D \in U_{\mathbb{C}}(\mathfrak{g}) .
$$

If $(\pi, \mathcal{H})$ is a continuous unitary representation and $v \in \mathcal{H}$ with $\varphi(g)=\langle\pi(g) v, v\rangle$ (the existence follows from the GNS construction), then [Ne10, Thm. 7.2] implies that $v$ is a smooth vector. Therefore the integrability of $\lambda$ is equivalent to the existence of a unitary representation $(\pi, \mathcal{H})$ and a smooth vector $v \in \mathcal{H}^{\infty}$ with

$$
\lambda(D)=\langle\mathrm{d} \pi(D) v, v\rangle \quad \text { for } \quad D \in U_{\mathbb{C}}(\mathfrak{g}) .
$$

The following proposition builds a bridge between analytic vectors and analytic functionals.

Proposition 6.3 Let $(\rho, \mathcal{D})$ be a strongly continuous $*$-representation of $\mathfrak{g}$ on the preHilbert space $\mathcal{D}$ and $\rho: U_{\mathbb{C}}(\mathfrak{g}) \rightarrow \operatorname{End}(\mathcal{D})$ its canonical extension. For $v \in \mathcal{D}$, the positive functional $\beta(D):=\langle\rho(D) v, v\rangle$ is analytic if and only if $v$ is an analytic vector. This implies that $v$ is an analytic vector for every operator $\rho(x), x \in \mathfrak{g}$, and that $\sum_{n=0}^{\infty} \frac{\left\|\rho(x)^{n} v\right\|}{n !}<\infty$ for $p(x)<R_{\beta, p} / 2$. 
Proof. First we observe that, for $x \in \mathfrak{g}$, we have

$$
\left\|\rho(x)^{n} v\right\|^{2}=\left\langle\rho(x)^{n} v, \rho(x)^{n} v\right\rangle=(-1)^{n} \beta\left(x^{2 n}\right) .
$$

If $\beta$ is analytic, then Proposition 3.4 provides an estimate of the form $\left\|\beta_{n}^{s}\right\|_{p} \leq C n ! t^{-n}$ for every $t<R_{\beta, p}$. This leads to

$$
\frac{\left\|\rho(x)^{n} v\right\|}{n !} \leq \sqrt{C} \frac{\sqrt{(2 n) !}}{n !} p(x)^{n} t^{-n} .
$$

Therefore $\frac{\sqrt{(2 n+2)(2 n+1)}}{n+1} \rightarrow 2$ implies that $\sum_{n=0}^{\infty} \frac{\left\|\rho(x)^{n} v\right\|}{n !}$ converges for $p(x)<t / 2$, hence for $p(x)<R_{\beta, p} / 2$.

Suppose, conversely, that $\sum_{n=0}^{\infty} \frac{\left\|\rho(x)^{n} v\right\|}{n !}$ converges for every $x$ in a 0-neighborhood $U$ of $\mathfrak{g}$. Then the series

$$
\left\langle\sum_{n=0}^{\infty} \frac{\rho(x)^{n} v}{n !}, v\right\rangle=\sum_{n=0}^{\infty} \frac{\left\langle\rho(x)^{n} v, v\right\rangle}{n !}=\sum_{n=0}^{\infty} \frac{\beta\left(x^{n}\right)}{n !}
$$

converges for every $x \in U$.

To see that $v$ is an analytic vector for $\rho(x)$, pick $t>0$ such that $t x \in U$ and observe that the series

$$
\sum_{n=0}^{\infty} \frac{\left\|\rho(x)^{n} v\right\| t^{n}}{n !}=\sum_{n=0}^{\infty} \frac{\left\|\rho(t x)^{n} v\right\|}{n !}
$$

converges.

Definition 6.4 (cf. [Ne00, Thm. III.1.3], Po71, Thm. 6.3], [Sch90, Thm. 8.6.2]) Let $\lambda \in U_{\mathbb{C}}(\mathfrak{g})^{*}$ be a positive analytic functional. On the dual space $U_{\mathbb{C}}(\mathfrak{g})^{*}$ we consider the right regular representation of $U_{\mathbb{C}}(\mathfrak{g})$ by $D \beta:=\beta \circ \rho_{D}$. Then there exists a unique Hilbert space $\mathcal{H}_{\lambda} \subseteq U_{\mathbb{C}}(\mathfrak{g})^{*}$ containing $\lambda$ for which the subspace

$$
\mathcal{D}_{\lambda}:=U_{\mathbb{C}}(\mathfrak{g}) \lambda
$$

is dense and on which the scalar product satisfies

$$
\beta(D)=\left\langle\beta, D^{*} \lambda\right\rangle \quad \text { for } \quad \beta \in \mathcal{H}_{\lambda}, D \in U_{\mathbb{C}}(\mathfrak{g}) .
$$

In particular, we have

$$
\left\langle D_{1} \lambda, D_{2} \lambda\right\rangle=\lambda\left(D_{2}^{*} D_{1}\right) .
$$

Now $\rho_{\lambda}(D) \beta:=D \beta:=\beta \circ \rho_{D}$ defines a $*$-representation of $U_{\mathbb{C}}(\mathfrak{g})$ on $\mathcal{D}_{\lambda}$ by unbounded operators, and for each $x \in \mathfrak{g}$, the operator $\rho_{\lambda}(x)$ on $\mathcal{D}_{\lambda}$ is skew-hermitian.

Proposition 6.5 If $\lambda$ is a positive analytic functional on $U_{\mathbb{C}}(\mathfrak{g})$, then the following assertions hold:

(a) $\lambda \in \mathcal{D}_{\lambda}$ is an analytic vector for every $\rho_{\lambda}(x), x \in \mathfrak{g}$. 
(b) If $\lambda$ is p-analytic for a submultiplicative continuous seminorm $p$ on $\mathfrak{g}$, then $\mathcal{D}_{\lambda}=$ $\rho_{\lambda}\left(U_{\mathbb{C}}(\mathfrak{g})\right) \lambda$ is equianalytic. More precisely, the series $\sum_{n=0}^{\infty} \frac{\rho_{\lambda}(x)^{n} v}{n !}$ converges for $p(x)<R_{\lambda, p} / 2$ and $v \in \mathcal{D}_{\lambda}$.

Proof. (a) In view of Proposition 6.3, this follows from $\lambda(D)=\left\langle\rho_{\lambda}(D) \lambda, \lambda\right\rangle$ for the representation $\left(\rho_{\lambda}, \mathcal{D}_{\lambda}\right)$. That this representation is strongly continuous follows from the continuity of the quadratic maps $x \mapsto\left\langle\rho_{\lambda}(x)^{2} \rho_{\lambda}(D) \lambda, \rho_{\lambda}(D) \lambda\right\rangle=\lambda\left(D^{*} x^{2} D\right)$ (Lemma 4.5).

(b) Let $v:=\rho_{\lambda}(D) \lambda$ for some $D \in U_{\mathbb{C}}(\mathfrak{g})$ and define

$$
\beta\left(D^{\prime}\right):=\left\langle\rho_{\lambda}\left(D^{\prime}\right) v, v\right\rangle=\lambda\left(D^{*} D^{\prime} D\right) \quad \text { for } \quad D^{\prime} \in U_{\mathbb{C}}(\mathfrak{g}) .
$$

Then Theorem 3.6 implies that $\beta$ is an analytic functional whose radius of convergence satisfies $R_{\beta, p} \geq r:=R_{\lambda, p}$. Therefore Proposition 6.3 implies that $v$ is an analytic vector for every operator $\rho_{\lambda}(x), x \in \mathfrak{g}$, and the corresponding exponential series converges for $p(x)<R_{\lambda, p} / 2 \leq R_{\beta, p} / 2$.

Definition 6.6 Let $(\rho, \mathcal{D})$ be a $*$-representation of $U_{\mathbb{C}}(\mathfrak{g})$ on the pre-Hilbert space $\mathcal{D}$. We call a subset $E \subseteq \mathcal{D}$ equianalytic if there exists a 0 -neighborhood $U \subseteq \mathfrak{g}$ such that

$$
\sum_{n=0}^{\infty} \frac{\left\|\rho(x)^{n} v\right\|}{n !}<\infty \quad \text { for } \quad v \in E, x \in U
$$

This implies in particular that each $v \in E$ is an analytic vector for every $\rho(x), x \in \mathfrak{g}$.

Lemma 6.7 Let $(\rho, \mathcal{D})$ be a *-representation of the BCH-Lie algebra $\mathfrak{g}$ on the dense equianalytic subspace $\mathcal{D}$ of $\mathcal{H}$. Then the closures $\overline{\rho(x)}, x \in \mathfrak{g}$, generate unitary oneparameter groups and there exists a 0-neighborhood $U \subseteq \mathfrak{g}$ on which the BCH product * is defined, and the map $\widetilde{\rho}: \mathfrak{g} \rightarrow \mathrm{U}(\mathcal{H}), x \mapsto e^{\overline{\rho(x)}}$ satisfies

$$
\widetilde{\rho}(x * y)=\widetilde{\rho}(x) \widetilde{\rho}(y) \quad \text { for } \quad x, y \in U .
$$

Proof. Nelson's Theorem 4.8 implies that the operators $\rho(x)$ are essentially skewadjoint, so that their closures generate unitary one-parameter groups and $\widetilde{\rho}$ is defined.

Let $U_{1} \subseteq \mathfrak{g}$ be an open balanced 0-neighborhood with the property that the function

$$
U_{1} \rightarrow \mathcal{H}, \quad x \mapsto \widetilde{\rho}(x)=\sum_{n=0}^{\infty} \frac{\rho(x)^{n}}{n !}
$$

is analytic for every $v \in \mathcal{D}$ (Definition [6.6). Let $U_{2} \subseteq U_{1}$ be a smaller convex 0neighborhood for which the BCH-multiplication defines an analytic map

$$
U_{2} \times U_{2} \rightarrow U_{1}, \quad(x, y) \mapsto x+y+\frac{1}{2}[x, y]+\cdots .
$$

Then we have for $v \in \mathcal{D}$ two analytic functions

$$
F_{1 / 2}: U_{2} \times U_{2} \rightarrow \mathbb{C}, \quad F_{1}(x, y)=\langle\widetilde{\rho}(y) v, \widetilde{\rho}(-x) v\rangle, \quad F_{2}(x, y)=\langle\widetilde{\rho}(x * y) v, v\rangle .
$$


To show that they coincide, it suffices to verify that their Taylor polynomials in $(0,0)$ agree. Let $\beta(D):=\langle\rho(D) v, v\rangle$. Then, for $F_{1}$, the homogeneous term of degree $(m, n)$ is given by

$$
T_{m, n}\left(F_{1}\right)(x, y)=\frac{1}{m ! n !}\left\langle\rho_{\lambda}(y)^{n} v, \rho_{\lambda}(-x)^{m} v\right\rangle=\frac{1}{m ! n !}\left\langle\rho_{\lambda}\left(x^{m} y^{n}\right) v, v\right\rangle=\beta\left(\frac{x^{m} y^{n}}{m ! n !}\right) .
$$

For $F_{2}$ the homogeneous term of degree $(m, n)$ has the form

$$
\sum_{k \leq n+m} \frac{1}{k !} T_{m, n}\left(\left\langle\rho_{\lambda}(x * y)^{k} v, v\right\rangle\right)=\beta\left(\sum_{k \leq n+m} \frac{1}{k !} T_{m, n}\left((x * y)^{k}\right)\right) .
$$

From the identity $e^{x} e^{y}=e^{x * y}$ in the completed free associative algebra $\mathcal{A}$ with two generators $x$ and $y$ we derive that

$$
\frac{x^{m} y^{n}}{m ! n !}=\sum_{k \leq n+m} \frac{1}{k !} T_{m, n}\left((x * y)^{k}\right)
$$

holds in $\mathcal{A}$, and this implies the corresponding relation in $U(\mathfrak{g})$ ([Bou89, Ch. 2, §6]). We conclude that

$$
\langle\widetilde{\rho}(x) \widetilde{\rho}(y) v, v\rangle=\langle\widetilde{\rho}(x * y) v, v\rangle \quad \text { for } \quad x, y \in U_{2}, v \in \mathcal{D} .
$$

Now polarization leads to

$$
\langle\widetilde{\rho}(x) \widetilde{\rho}(y) v, w\rangle=\langle\widetilde{\rho}(x * y) v, w\rangle \quad \text { for } \quad v, w \in \mathcal{D} .
$$

Since $\mathcal{D}$ is dense in $\mathcal{H}$, we obtain (11) for $U:=U_{2}$.

The following theorem is our first main result on integrability of Lie algebra representations based on a sufficient supply of analytic vectors.

Theorem 6.8 (Integrability Theorem) Let $G$ be a 1-connected BCH-Lie group with Lie algebra $\mathfrak{g}$ and $(\rho, \mathcal{D})$ be a $*$-representation of $\mathfrak{g}$ on the dense subspace $\mathcal{D}$ of $\mathcal{H}$. Assume that either

(a) $G$ is Banach and $\mathcal{D}$ consists of analytic vectors, or

(b) $\mathcal{D}$ is equianalytic.

Then there exists a unique unitary representation $(\pi, \mathcal{H})$ of $G$ on the corresponding Hilbert space $\mathcal{H}$ with $\left.\mathrm{d} \pi(x)\right|_{\mathcal{D}}=\rho(x)$ for $x \in \mathfrak{g}$.

Proof. With Nelson's Theorem 4.8 we see that the operators $\rho(x), x \in \mathfrak{g}$, are essentially skew-adjoint, so that their closures generate unitary one-parameter groups. This leads to a map $\widetilde{\rho}: \mathfrak{g} \rightarrow \mathrm{U}(\mathcal{H}), x \mapsto e^{\overline{\rho(x)}}$. We claim that

$$
\widetilde{\rho}(x * y)=\widetilde{\rho}(x) \widetilde{\rho}(y)
$$


holds for $x, y$ in some open 0 -neighborhood $U \subseteq \mathfrak{g}$. It suffices to show that

$$
\widetilde{\rho}(x * y) v=\widetilde{\rho}(x) \widetilde{\rho}(y) v \quad \text { for every } \quad v \in \mathcal{D} .
$$

(a) Suppose that $G$ is Banach. Let $\mathcal{H}_{v}^{0}:=\rho\left(U_{\mathbb{C}}(\mathfrak{g})\right) v$ and $\mathcal{H}_{v}$ be the closure of this subspace in $\mathcal{H}$. Then $\lambda(D):=\langle\rho(D) v, v\rangle$ is a positive analytic functional on $U_{\mathbb{C}}(\mathfrak{g})$ and the map

$$
\Gamma: \mathcal{H}_{v}^{0} \rightarrow U_{\mathbb{C}}(\mathfrak{g})^{*}, \quad \Gamma(w)(D):=\langle\rho(D) w, v\rangle
$$

is equivariant with respect to the right regular representation on $U_{\mathbb{C}}(\mathfrak{g})^{*}$ and satisfies $\Gamma\left(\mathcal{H}_{v}^{0}\right)=\rho_{\lambda}\left(U_{\mathbb{C}}(\mathfrak{g})\right) \lambda=\mathcal{D}_{\lambda}$. From $\left\langle D_{1} v, D_{2} v\right\rangle=\lambda\left(D_{2}^{*} D_{1}\right)$ we further derive that $\Gamma$ is isometric. Therefore Proposition 6.5(b) implies that $\mathcal{H}_{v}^{0}$ is equianalytic. It therefore suffices to consider case (b) to prove (13).

(b) If $\mathcal{D}$ is equianalytic, then Lemma 6.7 applies and provides an open 0-neighborhood $U \subseteq \mathfrak{g}$ with (12). Now we derive from Bou89, Ch. 3, §6, Lemma 1.1] (for Banach-Lie groups) and from GN10 (for BCH-Lie groups) that there exists a unique homomorphism $\pi: G \rightarrow \mathrm{U}(\mathcal{H})$ such that $\pi\left(\exp _{G} x\right)=\widetilde{\rho}(x)$ holds for all elements $x$ in some 0-neighborhood of $\mathfrak{g}$. This relation implies in particular that, for each $x \in \mathfrak{g}$, the unitary one-parameter group $\pi(\exp t x)$ is generated by the closure of the operator $\rho(x)$, so that $\pi$ is uniquely determined by the representation $\rho$ of $\mathfrak{g}$ on $\mathcal{D}$.

Lemma 6.9 If $\lambda$ is a positive analytic functional and $U \subseteq \mathfrak{g}$ a 0 -neighborhood, then $\widetilde{\rho}_{\lambda}(U) \lambda$ is total in $\mathcal{H}_{\lambda}$.

Proof. Let $\mathcal{K}$ denote the closure of the subspace generated by $\widetilde{\rho}(U) \lambda$. From

$$
\widetilde{\rho}_{\lambda}(x) \lambda=\sum_{n=0}^{\infty} \frac{1}{n !} \rho_{\lambda}(x)^{n} \lambda
$$

for $x$ sufficiently close to 0 we derive that $\rho_{\lambda}(x)^{n} \lambda=\left.\frac{d}{d t^{n}}\right|_{t=0} \widetilde{\rho}_{\lambda}(t x) \lambda \in \mathcal{K}$. Now the assertion follows from the fact that $U_{\mathbb{C}}(\mathfrak{g})$ is spanned by the elements of the form $x^{n}$, $x \in \mathfrak{g}, n \in \mathbb{N}_{0}$.

If $(\pi, \mathcal{H})$ is a unitary representation of the analytic Lie group $G$ and $v \in \mathcal{H}^{\omega}$ an analytic vector, then the corresponding functional $\pi^{v, v}(D):=\langle\mathrm{d} \pi(D) v, v\rangle$ on $U_{\mathbb{C}}(\mathfrak{g})$ is positive and analytic. The following theorem provides a converse which is known for finite dimensional groups (cf. Go69, Prop. 2.3]). Its strength lies in the fact that the information about the whole representation $\left(\pi_{\lambda}, \mathcal{H}_{\lambda}\right)$ is encoded completely on the infinitesimal level in the analytic functional $\lambda$.

Theorem 6.10 (Integrability Theorem for analytic functionals) Let $G$ be a 1-connected $B C H$-Lie group with Lie algebra $\mathfrak{g}$ and $\lambda \in U_{\mathbb{C}}(\mathfrak{g})^{*}$ be a positive analytic functional. Suppose that $U(\mathfrak{g}) \lambda \subseteq \operatorname{Hom}(U(\mathfrak{g}), \mathbb{C})_{\omega, p, r}$ for some $p \in \mathcal{P}(\mathfrak{g})$ and $r>0$, which is automatically satisfied if $\lambda \in \operatorname{Hom}(U(\mathfrak{g}), \mathbb{C})_{\omega, p}$ for some submultiplicative $p$. Then there exists a unique unitary representation $\left(\pi_{\lambda}, \mathcal{H}_{\lambda}\right)$ of $G$ for which the dense subspace $\mathcal{D}_{\lambda}$ consists of analytic vectors and

$$
\left.\mathrm{d} \pi_{\lambda}(x)\right|_{\mathcal{D}_{\lambda}}=\rho_{\lambda}(x) \quad \text { for } \quad x \in \mathfrak{g} .
$$


Proof. If $\lambda \in \operatorname{Hom}(U(\mathfrak{g}), \mathbb{C})_{\omega, p}$ for a submultiplicative seminorm $p$, then Theorem [3.6 implies that $\mathcal{D}_{\lambda}=U_{\mathbb{C}}(\mathfrak{g}) \lambda \subseteq \operatorname{Hom}(U(\mathfrak{g}), \mathbb{C})_{\omega, p, r}$ for some $r>0$. We assume that this condition is satisfied. Then

$$
U_{1}:=\{x \in \mathfrak{g}: p(x)<r / 2\}
$$

is an open convex 0 -neighborhood on which the series $\sum_{n=0}^{\infty} \frac{1}{n !} \rho_{\lambda}(x)^{n} v$ converges (Proposition 6.3). In particular, $\mathcal{D}_{\lambda}$ is equianalytic and Theorem [6.8(b) applies.

Specializing to Banach-Lie groups, the preceding theorem simplifies to:

Corollary 6.11 Let $G$ be a 1-connected Banach-Lie group with Lie algebra $\mathfrak{g}$ and $\lambda \in U_{\mathbb{C}}(\mathfrak{g})^{*}$ be a positive analytic functional. Then there exists a unique unitary representation $\left(\pi_{\lambda}, \mathcal{H}_{\lambda}\right)$ of $G$ for which the dense subspace $\mathcal{D}_{\lambda}$ consists of analytic vectors and

$$
\left.\mathrm{d} \pi_{\lambda}(x)\right|_{\mathcal{D}_{\lambda}}=\rho_{\lambda}(x) \quad \text { for } \quad x \in \mathfrak{g} .
$$

\section{Integrability without a given group}

Since not every Banach-Lie algebra is the Lie algebra of a Lie group ([EK64]), it is natural to ask to which extent the assumption of the existence of $G$ in Theorem 6.8 is needed to integrate the Lie algebra representation. The following theorem provides a corresponding variant, where no a priori given group is needed (cf. AN08 for a non-linear variant of this theorem).

Theorem 6.12 (Second Integrability Theorem) Let $\mathfrak{g}$ be a BCH-Lie algebra and $(\rho, \mathcal{D})$ be a faithful strongly continuous $*$-representation of $\mathfrak{g}$ on the pre-Hilbert space $\mathcal{D}$ by skew-adjoint operators. Assume that $\mathcal{D}$ consists of analytic vectors, so that the closure of each operator $\rho(x)$ generates a unitary one-parameter group on the completion $\mathcal{H}$ of $\mathcal{D}$. Assume that the subgroup

$$
\Gamma:=\left\{z \in \mathfrak{z}(\mathfrak{g}): e^{\overline{\rho(z)}}=\mathbf{1}\right\}
$$

of the center $\mathfrak{z}(\mathfrak{g})$ of $\mathfrak{g}$ is discrete. Then there exists a connected Lie group $G$ and a continuous unitary representation $(\pi, \mathcal{H})$ of $G$ on the corresponding Hilbert space $\mathcal{H}$ with $\left.\mathrm{d} \pi(x)\right|_{\mathcal{D}}=\rho(x)$ for $x \in \mathfrak{g}$.

Proof. With the same argument as in the proof of Theorem 6.8 we obtain a map $\widetilde{\rho}: \mathfrak{g} \rightarrow \mathrm{U}(\mathcal{H})$ satisfying $\widetilde{\rho}(x * y)=\widetilde{\rho}(x) \widetilde{\rho}(y)$ for $x, y$ in some open balanced 0 -neighborhood $U \subseteq \mathfrak{g}$. Shrinking $U$, we may even assume that

$$
\widetilde{\rho}(x * y * z)=\widetilde{\rho}(x) \widetilde{\rho}(y) \widetilde{\rho}(z) \quad \text { for } \quad x, y, z \in U .
$$

For $z=-x$ this leads to

$$
\widetilde{\rho}\left(e^{\operatorname{ad} x} y\right)=\widetilde{\rho}(x * y *(-x))=\widetilde{\rho}(x) \widetilde{\rho}(y) \widetilde{\rho}(-x)=\widetilde{\rho}(x) \widetilde{\rho}(y) \widetilde{\rho}(x)^{-1},
$$

and by passing to the infinitesimal generators, we obtain

$$
\rho\left(e^{\operatorname{ad} x} y\right)=\widetilde{\rho}(x) \rho(y) \widetilde{\rho}(x)^{-1} .
$$


We consider the subgroup $G:=\langle\widetilde{\rho}(\mathfrak{g})\rangle \subseteq \mathrm{U}(\mathcal{H})$ generated by $\widetilde{\rho}(\mathfrak{g})$. If $x \in U$ satisfies $\widetilde{\rho}(x)=\mathbf{1}$, then the preceding argument, together with the injectivity of $\rho$ implies that $e^{\operatorname{ad} x}=\operatorname{id}_{\mathfrak{g}}$. Since $\mathfrak{z}(\mathfrak{g})$ is open in $\left\{x \in \mathfrak{g}: e^{\operatorname{ad} x}=\operatorname{id}_{\mathfrak{g}}\right\}$ (cf. the Adjoint Integrability Theorem in GN10), we may shrink $U$ in such a way that, for $x \in U$, the relation $e^{\text {ad } x}=\operatorname{id}_{\mathfrak{g}}$ implies $x \in \mathfrak{z}(\mathfrak{g})$. In view of the discreteness of $\Gamma$, we may further shrink $U$ so that

$$
\widetilde{\rho}^{-1}(\mathbf{1}) \cap U=\{0\} .
$$

If $V \subseteq U$ is an open balanced 0-neighborhood with $V * V \subseteq U$, then $\left.\widetilde{\rho}\right|_{V}$ is injective. In fact, if $\widetilde{\rho}(x)=\widetilde{\rho}(y)$ for $x, y \in V$, then

$$
\widetilde{\rho}((-x) * y)=\widetilde{\rho}(-x) \widetilde{\rho}(y)=\widetilde{\rho}(x)^{-1} \widetilde{\rho}(y)=\mathbf{1}
$$

leads to $(-x) * y \in \widetilde{\rho}^{-1}(\mathbf{1}) \cap U=\{0\}$, We conclude that

$$
x=x * 0=x *((-x) * y)=(x *(-x)) * y=0 * y=y
$$

and hence that $\left.\widetilde{\rho}\right|_{V}$ is injective.

This means that $\widetilde{\rho}(V) \subseteq G$ carries a manifold structure for which $\left.\widetilde{\rho}\right|_{V}$ is a diffeomorphism, and that multiplication and inversion are smooth in an identity neighborhood in $V$. Since $G$ is generated by the image of every 0 -neighborhood in $V$, it carries a unique Lie group structure for which $\widetilde{\rho}$ defines a chart in a neighborhood of 0 (GN10, Thm. 3.3.2, Rem. 3.3.3]).

The curves $\gamma_{x}: \mathbb{R} \rightarrow G$ defined by $\gamma_{x}(t):=\widetilde{\rho}(t x)$ define smooth 1-parameter groups with $\gamma_{x}^{\prime}(0)=x$, where we identify $\mathfrak{g}$ via $T_{0}(\widetilde{\rho})$ with $T_{\mathbf{1}}(G)$. Therefore $\widetilde{\rho}$ is an exponential function of $G$. In particular, $G$ is locally exponential.

For every vector $v \in \mathcal{D}$, the map $x \mapsto \widetilde{\rho}(x) v$ is analytic in a 0-neighborhood, and since $\widetilde{\rho}$ is a local diffeomorphism, the vector $v$ has a smooth orbit map. For the unitary representation $(\pi, \mathcal{H})$ of $G$ given by the inclusion map $\pi: G \rightarrow \mathrm{U}(\mathcal{H})$, this means that it is smooth, hence in particular continuous. From the construction it follows that, for every $x \in \mathfrak{g}$, the generator $\overline{\mathrm{d} \pi}(x)$ of the unitary one-parameter group $t \mapsto \pi\left(\exp _{G}(t x)\right)$ restricts to $\rho(x)$ on $\mathcal{D}$.

Remark 6.13 For a strongly continuous representation $(\rho, V)$ of a Banach-Lie algebra $\mathfrak{g}$, the kernel is a closed ideal, so that $\mathfrak{g} / \operatorname{ker} \rho$ also is a Banach-Lie algebra. In the situation of Theorem 6.12, we may thus pass to an injective Lie algebra representation for which the theorem can be applied.

Here is an instructive example, showing that the group $\Gamma$ in Theorem 6.12 is not always discrete.

Example 6.14 Let $(X, \mathfrak{S}, \mu)$ be a probability space. For a measurable function $f: X \rightarrow \mathbb{C}$ we define

$$
\|f\|:=\sup \left\{\frac{\|f\|_{n}}{\sqrt[n]{n !}}: n \in \mathbb{N}\right\} \quad \text { for } \quad\|f\|_{n}:=\left(\int_{X}|f|^{n} d \mu\right)^{1 / n}
$$


Writing $\mathcal{M}(X, \mu)$ for the space of measurable functions $f: X \rightarrow \mathbb{C}$ modulo those vanishing on the complement of a zero set, we obtain a Banach space

$$
V:=\{f \in \mathcal{M}(X, \mu):\|f\|<\infty\}
$$

which can be viewed as a closed subspace of a weighted $\ell^{\infty}$-product of the spaces $L^{n}(X, \mu), n \in \mathbb{N}$.

For $\|f\|<\frac{1}{2}$ we have

$$
\sum_{n} \frac{\left\|f^{n}\right\|_{2}}{n !}=\sum_{n} \frac{\|f\|_{2 n}^{n}}{n !} \leq \sum_{n} \frac{\sqrt{(2 n) !}}{n !}\|f\|^{n}<\infty
$$

because $\frac{\sqrt{(2 n+2)(2 n+1)}}{n+1} \rightarrow 2$. Next we observe that for every measurable subset $E \subseteq X$ we have $\chi_{E} \in V$ because

$$
\left\|\chi_{E}\right\|=\sup _{n} \frac{\left\|\chi_{E}\right\|_{n}}{\sqrt[n]{n !}}=\sup _{n} \sqrt[n]{\frac{\mu(E)}{n !}}<\infty
$$

follows from $\mu(E) / n ! \rightarrow 0$. From

$$
(n !)^{n+1}=(n !)^{n} n ! \leq(n !)^{n}(n+1)^{n}=((n+1) !)^{n}
$$

we derive that $\sqrt[n]{n !} \leq \sqrt[n+1]{(n+1) !}$, i.e., the sequence $\sqrt[n]{n !}$ is increasing. The formula for the radius of convergence of the exponential series now yields $\sqrt[n]{n !} \rightarrow \infty$.

We claim that $\chi_{E_{k}} \rightarrow 0$ in $V$ if $\mu\left(E_{k}\right) \rightarrow 0$. Let $\varepsilon>0$ and $N \in \mathbb{N}$ with $\sqrt[N]{N !}>\varepsilon^{-1}$. Suppose that $\mu\left(E_{k}\right)^{1 / n} \leq \varepsilon$ for $k \geq N_{0}$ and every $n \leq N$. The relation

$$
\sqrt[n]{\frac{\mu\left(E_{k}\right)}{n !}} \leq \frac{1}{\sqrt[n]{n !}} \quad \text { for } \quad k \geq N_{0}, n>N
$$

and

$$
\sqrt[n]{\frac{\mu\left(E_{k}\right)}{n !}} \leq \frac{\varepsilon}{\sqrt[n]{n !}}<\varepsilon \quad \text { for } \quad k \geq N_{0}, n \leq N
$$

then leads to

$$
\left\|\chi_{E_{k}}\right\| \leq \sup _{n} \sqrt[n]{\frac{\mu\left(E_{k}\right)}{n !}} \leq \varepsilon \quad \text { for } \quad k \geq N_{0} .
$$

We conclude that $\chi\left(E_{k}\right) \rightarrow 0$ in $V$. Therefore the closed subgroup

$$
\Gamma:=\{f \in V: f(X) \subseteq 2 \pi \mathbb{Z}\}
$$

is not discrete.

The Banach space $V$ acts by unbounded multiplication operators on $L^{2}(X, \mu)$ :

$$
\rho(f) \xi:=i f \xi
$$


The definition of the norm in $V$ implies that each bounded function $\xi \in L^{2}(X, \mu)$ is an analytic vector for $V$ :

$$
\sum_{n} \frac{\left\|(i f)^{n} \xi\right\|_{2}}{n !} \leq\|\xi\|_{\infty} \sum_{n} \frac{\left\|f^{n}\right\|_{2}}{n !}<\infty \quad \text { for } \quad\|f\|<\frac{1}{2} .
$$

Therefore we have a $*$-representation $\left(L^{\infty}(X, \mu), \rho\right)$ of $V$ by unbounded operators on the dense subspace $L^{\infty}(X, \mu)$ of the Hilbert space $L^{2}(X, \mu)$, for which the group

$$
\left\{f \in \mathfrak{g}: e^{\rho(f)}=\mathrm{id}\right\}=\Gamma
$$

is not discrete.

Remark 6.15 The preceding example shows in particular that there exists an abelian Banach-Lie group $G$ and a continuous unitary representation $(\pi, \mathcal{H})$ for which $\mathcal{H}^{\omega}$ is dense but the closed subgroup $\operatorname{ker} \pi$ is not discrete and satisfies $\mathrm{E}(\operatorname{ker} \pi)=\{0\}$.

\section{Extension of local positive definite functions}

Definition 7.1 Let $G$ be a group and $U \subseteq G$ be a subset. A function $\varphi: U U^{-1} \rightarrow \mathbb{C}$ is said to be positive definite if the kernel $K: U \times U \rightarrow \mathbb{C},(x, y) \mapsto \varphi\left(x y^{-1}\right)$ is positive definite.

The main result of this section is Theorem 7.3 which asserts that any positive definite analytic function defined in a 1-neighborhood of a simply connected BCHLie group $G$ extends to an analytic function on $G$ which is positive definite. This is a quite remarkable result which is far from being true in the $C^{\infty}$-context. For a discussion of related extension problems for smooth positive definite functions on finite dimensional Lie groups we refer to Jo91 and the references given there. As we have seen in the introduction, for $G=\mathbb{R}^{n}$, such extension problems are part of the classical theory of moments. The corresponding version of Theorem 7.3 can be found in $\mathrm{Fa08}$, Prop. 3.10].

Before we turn to the extension theorem, we make the following observation on analytic functions on product spaces.

Lemma 7.2 Let $X, Y$ be locally convex spaces and $Z$ a Banach space. Let $U \subseteq X \times Y$ be an open neighborhood of $\left(x_{0}, y_{0}\right)$ and $f: U \rightarrow Z$ be an analytic map. Then there exists an open neighborhood $U_{x_{0}}$ of $x_{0}$ and an open neighborhood $U_{y_{0}}$ of $y_{0}$ such that for every $x \in U_{x_{0}}$ and $h \in U_{y_{0}}-y_{0}$ we have

$$
f\left(x, y_{0}+h\right)=\sum_{n=0}^{\infty} \frac{1}{n !}\left(\partial_{(0, h)}^{n} f\right)\left(x, y_{0}\right) .
$$

Proof. In view of [BS71b, Thm. 5.1,Prop. 5.4], we may assume that $f$ has a complex analytic extension $f: U_{\mathbb{C}} \rightarrow Z_{\mathbb{C}}$, where $U_{\mathbb{C}}$ is an open neighborhood of $\left(x_{0}, y_{0}\right)$ in the complex locally convex space $X_{\mathbb{C}} \times Y_{\mathbb{C}}$. Let $V_{x_{0}}$, resp., $V_{y_{0}}$ be an open balanced 
neighborhood of $x_{0}$ in $U_{\mathbb{C}}$, resp., $y_{0}$ in $Y_{\mathbb{C}}$ such that $V_{x_{0}} \times V_{y_{0}} \subseteq U_{\mathbb{C}}$. Then BS71b, Prop. 5.5] implies that

$$
f\left(x, y_{0}+h\right)=\sum_{n=0}^{\infty} \frac{1}{n !}\left(\partial_{(0, h)}^{n} f\right)\left(x, y_{0}\right) \quad \text { for } \quad x \in V_{x_{0}}, h \in V_{y_{0}}-y_{0} .
$$

Hence the assertion follows with $U_{x_{0}}:=V_{x_{0}} \cap X$ and $U_{y_{0}}:=V_{y_{0}} \cap Y$.

Theorem 7.3 (Extension of local positive definite analytic functions) Let $G$ be a simply connected Fréchet-BCH-Lie group, $V \subseteq G$ an open connected 1-neighborhood and $\varphi: V V^{-1} \rightarrow \mathbb{C}$ an analytic positive definite function. Then there exists a unique positive definite analytic function $\widetilde{\varphi}: G \rightarrow \mathbb{C}$ extending $\varphi$.

Proof. The uniqueness of $\widetilde{\varphi}$ follows from the connectedness of $G$ and the uniqueness of analytic continuation.

Step 1: To obtain its existence, we consider the reproducing kernel Hilbert space $\mathcal{H}_{K} \subseteq \mathbb{C}^{V}$ defined by the kernel $K$ via $f(g)=\left\langle f, K_{g}\right\rangle$ for $g \in V$ and $K_{g}(h)=K(h, g)$. Then the analyticity of the function

$$
V \times V \rightarrow \mathbb{C}, \quad(g, h) \mapsto\left\langle K_{h}, K_{g}\right\rangle=K(g, h)=\varphi\left(g h^{-1}\right)
$$

implies that the map $\eta: V \rightarrow \mathcal{H}_{K}, g \mapsto K_{g}$ is analytic (Theorem 5.1). Here we use that $G$ is assumed to be Fréchet. Hence all functions in $\mathcal{H}_{K}$ are analytic, and that we obtain for each $x \in \mathfrak{g}$ an operator on $\mathcal{H}_{K}^{0}:=\operatorname{span} \eta(V)$ by

$$
(\rho(x) f)(g):=\left.\frac{d}{d t}\right|_{t=0} f\left(g \exp _{G}(t x)\right)
$$

because $\left.\frac{d}{d t}\right|_{t=0} K_{y}\left(g \exp _{G}(t x)\right)$ exists for every $g \in V$ and $x \in \mathfrak{g}$.

Step 2: That we thus obtain a representation $\rho: \mathfrak{g} \rightarrow \operatorname{End}\left(\mathcal{H}_{K}^{0}\right)$ follows from the embedding $\mathcal{H}_{K}^{0} \hookrightarrow C^{\omega}(V, \mathbb{C})$ and the fact that $\mathfrak{g}$ acts by a Lie algebra of (left invariant) vector fields on this space. Next we observe that

$$
\begin{aligned}
& \left\langle\rho(x) K_{g}, K_{h}\right\rangle=\left.\frac{d}{d t}\right|_{t=0} K_{g}\left(h \exp _{G}(t x)\right)=\left.\frac{d}{d t}\right|_{t=0} K\left(h \exp _{G}(t x), g\right) \\
& =\left.\frac{d}{d t}\right|_{t=0} \varphi\left(h \exp _{G}(t x) g^{-1}\right)=\left.\frac{d}{d t}\right|_{t=0} K\left(h, g \exp _{G}(-t x)\right)=\left.\frac{d}{d t}\right|_{t=0} \overline{K_{h}\left(g \exp _{G}(-t x)\right)} \\
& =-\overline{\left(\rho(x) K_{h}\right)(g)}=-\overline{\left\langle\rho(x) K_{h}, K_{g}\right\rangle}=-\left\langle K_{g}, \rho(x) K_{h}\right\rangle .
\end{aligned}
$$

Therefore $\rho$ extends to a $*$-representation of $U_{\mathbb{C}}(\mathfrak{g})$ on $\mathcal{H}_{K}^{0}$.

Step 3: We claim that each $\eta(g)=K_{g}, g \in V$, is an analytic vector with

$$
\eta\left(g \exp _{G}(-x)\right)=\sum_{n=0}^{\infty} \frac{1}{n !} \rho(x)^{n} \eta(g)
$$


for $x$ sufficiently close to 0 . The function $x \mapsto \eta\left(g \exp _{G} x\right)$ is analytic on a 0 neighborhood of $\mathfrak{g}$ and

$$
\begin{aligned}
\left.\frac{d^{n}}{d t^{n}}\right|_{t=0}\left(\eta\left(g \exp _{G}(t x)\right)\right)(y) & =\left.\frac{d^{n}}{d t^{n}}\right|_{t=0} K_{g \exp _{G} t x}(y)=\left.\frac{d^{n}}{d t^{n}}\right|_{t=0} \varphi\left(y \exp _{G}(-t x) g^{-1}\right) \\
& =\left.\frac{d^{n}}{d t^{n}}\right|_{t=0} K_{g}\left(y \exp _{G}(-t x)\right)=\left(\rho(-x)^{n} \eta(g)\right)(y) .
\end{aligned}
$$

This leads to

$$
\left.\frac{d^{n}}{d t^{n}}\right|_{t=0} \eta\left(g \exp _{G}(-t x)\right)=\rho(x)^{n} \eta(g)
$$

and hence that (14) holds on a 0-neighborhood of $\mathfrak{g}$.

Step 4: Let $W_{G} \subseteq V$ be an open 1-neighborhood and $W_{\mathfrak{g}} \subseteq \mathfrak{g}$ an open balanced 0-neighborhood with $W_{G} \exp _{G}\left(W_{\mathfrak{g}}\right) \subseteq V$. Next we show that $\eta\left(W_{G}\right)$ is equianalytic and spans a dense subspace of $\mathcal{H}_{K}$.

Since the map $W_{G} \times W_{\mathfrak{g}} \rightarrow \mathcal{H}_{K},(g, x) \mapsto \eta\left(g \exp _{G}(x)\right)$ is analytic, Lemma 7.2 shows that, after shrinking $W_{G}$ and $W_{\mathfrak{g}}$, we may assume that

$$
\eta\left(g \exp _{G}(-x)\right)=\sum_{n=0}^{\infty} \frac{1}{n !} \rho(x)^{n} \eta(g) \quad \text { for } \quad g \in W_{G}, x \in W_{\mathfrak{g}} .
$$

This means that $\eta\left(W_{G}\right)$ is equianalytic. To see that $\mathcal{K}:=\overline{\operatorname{span}\left(\eta\left(W_{G}\right)\right)}$ is dense, we use the analyticity of $\eta$ to see that $\eta(V) \subseteq \mathcal{K}$, which shows that $\mathcal{H}_{K}=\mathcal{K}$.

Applying Theorem 6.8 with $\mathcal{D}=\operatorname{span}\left(\eta\left(W_{G}\right)\right)$, we now obtain a continuous unitary representation $\left(\pi, \mathcal{H}_{K}\right)$ with $\pi\left(\exp _{G}(x)\right)=e^{\overline{\rho(x)}}$ for every $x \in \mathfrak{g}$. Then

$$
\widetilde{\varphi}(g):=\left\langle\pi(g) K_{\mathbf{1}}, K_{\mathbf{1}}\right\rangle=\langle\pi(g) \varphi, \varphi\rangle
$$

is an analytic positive definite function on $G$, and for $x \in W_{\mathfrak{g}}$ we have

$$
\widetilde{\varphi}\left(\exp _{G} x\right)=\sum_{n=0}^{\infty} \frac{\left\langle\rho(x)^{n} \varphi, \varphi\right\rangle}{n !}=\left\langle\eta\left(\exp _{G}(-x)\right), \varphi\right\rangle=K_{\exp _{G}(-x)}(\mathbf{1})=\varphi\left(\exp _{G} x\right) .
$$

Therefore $\widetilde{\varphi}$ coincides with $\varphi$ in a 1-neighborhood, so that the analyticity of $\varphi$ and the connectedness of $V$ lead to $\left.\widetilde{\varphi}\right|_{V}=\varphi$.

Acknowledgement: We thank Stéphane Merigon for a careful reading of this paper and for several discussions on its subject matter. We are also grateful to J. Faraut for pointing out some crucial references.

\section{References}

[AN08] Abouqateb, A., and K.-H. Neeb, Integration of locally exponential Lie algebras of vector fields, Annals Global Analysis Geom. 33:1 (2008), 89-100

[Ak65] Akhiezer, N. I., "The Classical Moment Problem," Oliver and Boyd, Edinburgh, 1965 
[BN08] Beltita, D., and K.-H. Neeb, A non-smooth continuous unitary representation of a Banach-Lie group, J. Lie Theory 18 (2008), 933-936

[BCR84] Berg, C., J.P.R. Christensen, and P. Ressel, "Harmonic analysis on semigroups," Graduate Texts in Math. 100, Springer Verlag, Berlin, Heidelberg, New York, 1984

[BS71a] Bochnak, J., Siciak, J., Polynomials and multilinear mappings in topological vector spaces, Studia Math. 39 (1971), 59-76

[BS71b] -, Analytic functions in topological vector spaces, Studia Math. 39 (1971), $77-112$

[BY75] Borchers, H.-J., and Yngvason, J., Integral representations of Schwinger functionals and the moment problem over nuclear spaces, Comm. math. Phys. 43:3 (1975), 255-271

[Bou07] Bourbaki, N., "Espaces vectoriels topologiques. Chap.1 à 5", SpringerVerlag, Berlin, 2007

[Bou89] - , "Lie Groups and Lie Algebras, Chapter 1-3," Springer-Verlag, Berlin, 1989

[CD58] Cartier, P., and J. Dixmier, Vecteurs analytiques dans les représentations de groupes de Lie, Amer. J. Math. 80 (1958), 131-145

[DG08] Driver, B., and M. Gordina, Square integrable holomorphic functions on infinite-dimensional Heisenberg type groups, arXiv:math.PR.0809.4979v1

[EK64] van Est, W. T., and Th. J. Korthagen, Non enlargible Lie algebras, Proc. Kon. Ned. Acad. v. Wet. Series A, Indag. Math. 26 (1964), 15-31

[Fa08] Faraut, J., "Infinite Dimensional Spherical Analysis," COE Lectures Note Vol. 10, Kyushu Univ., 2008

[FSSS72] Flato, M., J. Simon, H. Snellman, and D. Sternheimer, Simple facts about analytic vectors and integrability, Ann. Sci. École Norm. Sup. (4) 5 (1972), 423-434

[Ga60] Gårding, L., Vecteurs analytiques dans les représentations des groupes de Lie, Bull. Soc. Math. France 88 (1960), 73-93

[Go69] Goodman, R. W., Analytic and entire vectors for representations of Lie groups, Trans. Amer. Math. Soc. 143 (1969), 55-76

[Gl02] Glöckner, H., Infinite-dimensional Lie groups without completeness restrictions, in "Geometry and Analysis on Finite and Infinite-dimensional Lie Groups," A. Strasburger, W. Wojtynski, J. Hilgert and K.-H. Neeb (Eds.), Banach Center Publications 55 (2002), 43-59 
[GN10] Glöckner, H., and K.-H. Neeb, "Infinite dimensional Lie groups, Vol. I, Basic Theory and Main Examples," book in preparation

[GJ83] Goodman, F., and P. E. T. Jørgensen, Lie algebras of unbounded derivations, J. Funct. Anal. 52 (1983), 369-384

[Heg72] Hegerfeldt, G. C., Gårding domains and analytic vectors for quantum fields, J. Math. Phys. 13 (1972), 821-827

[Heg75] - Extremal decompositions of Wightman functions and of states on nuclear *-algebras by Choquet theory, Comm. Math. Phys. 45:2 (1975), 133135

[Jo86] Jørgensen, P.E.T., Analytic continuation of local representations of Lie groups, Pac. J. Math. 125:2 (1986), 397-408

[Jo87] -, Analytic continuation of local representations of symmetric spaces, J. Funct. Anal. 70 (1987), 304-322

[Jo88] Jørgensen, P. E. T., "Operators and Representation Theory," Math. Studies 147, North -Holland, 1988

[Jo91] - Integral representations for locally defined positive definite functions on Lie groups, Int. J. Math. 2:3 (1991), 257-286

[Kr49] Krein, M. G., Hermitian positive definite kernels on homogeneous spaces I, Amer. Math. Soc. Transl. Ser. 2, Vol. 34, 69-108

[LM75] Lüscher, M., and G. Mack, Global conformal invariance and quantum field theory, Comm. Math. Phys. 41 (1975), 203-234

[Mag92] Magyar, M., "Continuous Linear Representations," North-Holland, Math. Studies 168, 1992

[Me10] Merigon, S., Integrating representations of Banach-Lie algebras, arXiv:math.RT.1003.0999v1, 4 Mar 2010

[Mil84] Milnor, J., Remarks on infinite-dimensional Lie groups, in DeWitt, B., Stora, R. (eds), "Relativité, groupes et topologie II" (Les Houches, 1983), North Holland, Amsterdam, 1984; 1007-1057

[Mo72] Moore, R. T., Measurable, continuous and smooth vectors for semigroup and group representations, Amer. Math. Soc. 19 (1972), 1-80

[MNS09] Müller, C., K.-H. Neeb, and H. Seppänen, Borel-Weil Theory for Root Graded Banach-Lie groups, Int. Math. Res. Notices, to appear; arxiv:math.RT.0903.1188

[Ne00] Neeb, K.-H., "Holomorphy and Convexity in Lie Theory," Expositions in Mathematics 28, de Gruyter Verlag, Berlin, 1999 
[Ne06] - Towards a Lie theory of locally convex groups, Jap. J. Math. 3rd ser. 1:2 (2006), 291-468

[Ne10] - On differentiable vectors for representations of infinite dimensional Lie groups, J. Funct. Anal., to appear; arXiv:math.RT.1002.1602, 21 Apr 2010

[Nel59] Nelson, E., Analytic vectors, Annals of Math. 70:3 (1959), 572-615

[O190] Olshanski, G. I., Unitary representations of infinite dimensional $(G, K)$ pairs and the formalism of $R$. Howe, in "Representations of Lie Groups and Related Topics," Eds. A. M. Vershik and D. P. Zhelobenko, Advanced Studies in Contemp. Math. 7, Gordon and Breach Science Publ., 1990

[O191] - On semigroups related to infinite dimensional groups, in "Topics in representation theory," Amer. Math. Soc., Adv. Sov. mathematics 2 (1991), $67-101$

[Po71] Powers, R.T., Self-adjoint algebras of unbounded operators, Comm. Math. Phys. 21 (1971), 85-124

[Po74] -, Selfadjoint algebras of unbounded operators, II, Trans. Amer. Math. Soc. 187:1 (1974), 261-293

[Re69] Reed., M. C., A Gårding domain for quantum fields, Comm. Math. Phys. 14 (1969), 336-346

[RS75] Reed, S., and B. Simon, "Methods of Modern Mathematical Physics II: Fourier Analysis, Self-adjointness," Academic Press, New York, 1975

[Ru73] Rudin, W., "Functional Analysis," McGraw Hill, 1973

[Sa91] Samoilenko, Y. S., "Spectral Theory of Families of Self-Adjoint Operators", Mathematics and its Applications (Soviet Series), Kluwer Acad. Publ., 1991

[Sch78] Schmüdgen, K., Positive cones in enveloping algebras, Reports Math. Phys. 14 (1978), 385-404

[Sch90] - "Unbounded Operator Algebras and Representation Theory," Operator Theory: Advances and Applications 37, Birkhäuser Verlag, Basel, 1990

[Sh96] Shiryaev, A. N., "Probability," 2nd Edition, Graduate Texts in Math. 95, Springer, 1996

[Si72] Simon, J., On the integrability of representations of finite dimensional real Lie algebras, Comm. Math. Phys. 28 (1972), 39-46

[Wa72] Warner, G., "Harmonic analysis on semisimple Lie groups I," Springer Verlag, Berlin, Heidelberg, New York, 1972 